\title{
Biomimetic Synthesis of Antimalarial Naphthoquinones
}

\author{
Jeremiah P. Malerich, Thomas J. Maimone, Gregory I. Elliott", and Dirk Trauner*
}

Center for New Directions in Organic Synthesis, Department of Chemistry, University of California Berkeley, Berkeley, California 94720-1460

trauner@cchem.berkeley.edu

${ }^{\dagger}$ G. I. Elliot: Department of Chemistry and The Skaggs Institute for Chemical Biology, The Scripps Research Institute, 10550 North Torrey Pines Road, La Jolla, California 92037

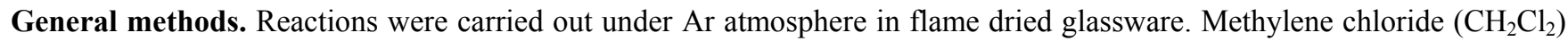
and toluene $(\mathrm{PhMe})$ were purified by passage over activated alumina. Tetrahydrofuran (THF) was distilled from $\mathrm{Na}$ /benzophenone immediately prior to use. Benzene $(\mathrm{PhH})$ and acetonitrile $(\mathrm{MeCN})$ were distilled from $\mathrm{CaH}_{2}$ immediately prior to use. All other reagents and solvents were used without further purification from commercial sources. Organic extracts were dried over $\mathrm{MgSO}_{4}$, unless otherwise specified. Flash column chromatography was carried out with EcoChrom ICN SiliTech 32-63 D $60 \AA$ silica gel. Reactions and chromatography fractions were monitored with Merck silica gel 60 $\mathrm{F}_{254}$ plates, visualized with ceric ammonium molybdate, $p$-anisaldehyde, and dinitrophenylhydrazine. NMR spectra were measured in $\mathrm{CDCl}_{3}$ unless otherwise specified and calibrated from residual solvent signal on a Bruker DRX spectrometer at $500 \mathrm{MHz}$ for ${ }^{1} \mathrm{H}$ spectra and $125 \mathrm{MHz}$ for ${ }^{13} \mathrm{C}$ spectra or a Bruker AVB spectrometer at $400 \mathrm{MHz}$ for ${ }^{1} \mathrm{H}$ spectra and 100 $\mathrm{MHz}$ for ${ }^{13} \mathrm{C}$ spectra. IR spectra were measured with a Genesis FT-IR spectrometer by thin film. Melting points were determined with an electrothermal apparatus and are uncorrected. Enantiomeric excess was determined by chiral analytical HPLC with a CHIRALPAK AD column $(250 \times 4.6 \mathrm{~mm})$ with a flow rate of $1.0 \mathrm{~mL} / \mathrm{min}$. Integration was done manually and the estimated error of e.e. is $\pm 1 \%$.

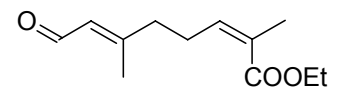

25

(Z)-2,6-Dimethyl-8-oxo-octa-2,6-dienoic acid ethyl ester (25). To a solution of alcohol 24 (1.16 $\mathrm{g}$, $5.464 \mathrm{mmol})$ in $\mathrm{CH}_{2} \mathrm{Cl}_{2}(25 \mathrm{~mL})$ was added $\mathrm{MnO}_{2}(4.59 \mathrm{~g}, 54.6 \mathrm{mmol})$. After $12 \mathrm{~h}$ the reaction mixture was filtered through Celite and concentrated in vacuo. Flash chromatography (4:1 hexanes : EtOAc) afforded unsaturated aldehyde 25 (1.04 g, $90 \%)$ as a pale yellow oil: $\mathrm{R}_{\mathrm{f}} 0.27$ (3:1 hexanes : EtOAc); IR: 2980, 2956, 2927, 2855, $1711,1674 \mathrm{~cm}^{-1} ;{ }^{1} \mathrm{H} \mathrm{NMR}(500 \mathrm{MHz}): \delta 9.98$ $(\mathrm{d}, J=7.9 \mathrm{~Hz}, 1 \mathrm{H}), 5.87(\mathrm{~m}, 2 \mathrm{H}), 4.18(\mathrm{q}, J=6.9 \mathrm{~Hz}, 2 \mathrm{H}), 2.68(\mathrm{q}, J=7.2 \mathrm{~Hz}, 2 \mathrm{H}), 2.31(2, J=7.3 \mathrm{~Hz}, 2 \mathrm{H}), 2.16(\mathrm{~s}, 3 \mathrm{H})$, $1.88(\mathrm{~s}, 3 \mathrm{H}), 1.28(\mathrm{t}, J=6.9 \mathrm{~Hz}, 3 \mathrm{H}) ;{ }^{13} \mathrm{C}$ NMR $(125 \mathrm{MHz}) \delta 191.2,167.7,162.9,140.3,128.6,127.5,60.2,39.9,26.9,20.6$, 17.4, 14.2; HRMS (EI+) Calcd for $\mathrm{C}_{12} \mathrm{H}_{18} \mathrm{O}_{3}\left(\mathrm{M}^{+}\right): 210.1256$, Found: 210.1252 .

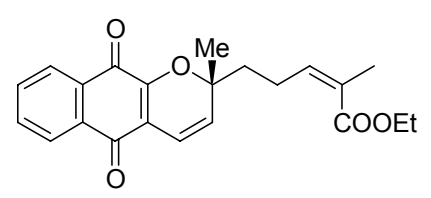

27

(Z)-2-Methyl-5-(2-methyl-5,10-dioxo-5,10-dihydro-2H-benzo[g]chromen-2-yl)-pent-2-enoic acid ethyl ester (27). A solution of 2-hydroxynaphthoquinone $(1.47 \mathrm{~g}, 8.44 \mathrm{mmol})$, aldehyde 25 (591 mg, $2.81 \mathrm{mmol}), \beta$-alanine (38 mg, 0.42 mmol), and HOAc $(0.2 \mathrm{~mL})$ in $\mathrm{PhH}(100 \mathrm{~mL})$ was heated to reflux. After $3 \mathrm{~h}$, the reaction mixture was concentrated. Flash chromatography $\left(3: 1\right.$ hexanes : EtOAc) afforded chromene $27(521 \mathrm{mg}, 51 \%)$ as a red oil: $\mathrm{R}_{\mathrm{f}} 0.59$ (1:1 hexanes : EtOAc); IR: 2979, 2928, 1709, 1675, 1651, 1595, $1571 \mathrm{~cm}^{-1} ;{ }^{1} \mathrm{H}$ NMR $(500 \mathrm{MHz}): \delta 8.09(\mathrm{~m}, 2 \mathrm{H}), 7.69(\mathrm{~m}, 2 \mathrm{H}), 6.70(\mathrm{~d}, J=10.2$ $\mathrm{Hz}, 1 \mathrm{H}), 5.93(\mathrm{dt}, J=1.1,7.5 \mathrm{~Hz}, 1 \mathrm{H}), 5.69(\mathrm{~d}, J=10.2 \mathrm{~Hz}, 1 \mathrm{H}), 4.11(\mathrm{~m}, 2 \mathrm{H}), 2.62(\mathrm{~m}, 2 \mathrm{H}), 2.00-2.08(\mathrm{~m}, 2 \mathrm{H}), 1.82(\mathrm{~m}$, $4 \mathrm{H}), 1.52(\mathrm{~s}, 3 \mathrm{H}), 1.23(\mathrm{t}, J=7.2 \mathrm{~Hz}, 3 \mathrm{H}) ;{ }^{13} \mathrm{C} \mathrm{NMR}(125 \mathrm{MHz}) \delta 181.8,179.8,167.7,152.6,141.6,133.9,133.2,131.6$, $131.5,129.5,127.9,126.2,126.1,117.5,116.3,83.0,60.1,41.0,27.6,24.5,20.6,14.2$; HRMS (EI+) Calcd for $\mathrm{C}_{22} \mathrm{H}_{22} \mathrm{O}_{5}$ $\left(\mathrm{M}^{+}\right): 366.1467$, Found: 366.1473 . 


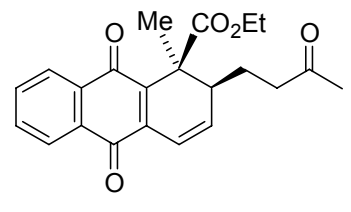

29

1-Methyl-9,10-dioxo-2-(3-oxo-butyl)-1,2,9,10-tetrahydro-anthracene-1-carboxylic acid ethyl ester (29). A solution of $27(50 \mathrm{mg}, 0.137 \mathrm{mmol})$ in $\mathrm{PhH}(10 \mathrm{~mL})$ was heated to $160^{\circ} \mathrm{C}$ for $12 \mathrm{~h}$. After concentration of the reaction mixture, flash chromatography yielded $29(41 \mathrm{mg}, 82 \%)$ as a yellow solid: $\mathrm{mp} 135-136{ }^{\circ} \mathrm{C} ; \mathrm{R}_{\mathrm{f}} 0.40(1: 1$ hexanes : EtOAc); IR: 2985 , 2933, 1728, 1661, $1592 \mathrm{~cm}^{-1}$; ${ }^{1} \mathrm{H}$ NMR (500 MHz): $\delta$ 8.08-8.12 (m, 1H), 8.01-8.05 (m, 1H), $7.72(\mathrm{~m}, 2 \mathrm{H}), 6.90(\mathrm{~d}, J=9.8$ $\mathrm{Hz}, 1 \mathrm{H}), 6.34(\mathrm{dd}, J=6.0,9.8 \mathrm{~Hz}, 1 \mathrm{H}), 4.20(\mathrm{~m}, 2 \mathrm{H}), 2.52(\mathrm{~m}, 2 \mathrm{H}), 2.36(\mathrm{~m}, 1 \mathrm{H}), 2.07(\mathrm{~s}, 3 \mathrm{H}), 1.72(\mathrm{~m}, 1 \mathrm{H}), 1.58-1.67(\mathrm{~m}$, $1 \mathrm{H}), 1.47(\mathrm{~s}, 3 \mathrm{H}), 1.22(\mathrm{t}, J=7.2 \mathrm{~Hz}, 3 \mathrm{H}) ;{ }^{13} \mathrm{C}$ NMR $(125 \mathrm{MHz}) \delta 207.9,183.6,183.1,173.7,142.3,136.4,135.7,133.9$, 133.4, 133.0, 131.4, 126.3, 126.2, 118.5, 61.0, 47.5, 43.2, 39.7, 29.9, 26.7, 23.0, 14.0.; HRMS (FAB+) Calcd for $\mathrm{C}_{22} \mathrm{H}_{23} \mathrm{O}_{5}$ $(\mathrm{M}+\mathrm{H})^{+}: 367.1545$, Found: 367.1538 .

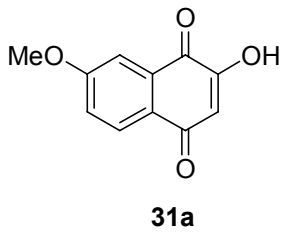

2-Hydroxy-6-methoxy-[1,4]naphthoquinone (31a). Oxygen was passed through a solution of $\mathrm{KO} t \mathrm{Bu}(50 \mathrm{~g}, 446 \mathrm{mmol})$ in $\mathrm{HO} t \mathrm{Bu}(500 \mathrm{~mL})$ for $30 \mathrm{~min}$. 6-Methoxytetralone (30a) $(10.0 \mathrm{~g}, 56.8 \mathrm{mmol})$ was added, and oxygen continued to bubble through the reaction mixture for $4 \mathrm{~h}$. The mixture was cooled to $0{ }^{\circ} \mathrm{C}$, quenched with $1 \mathrm{M} \mathrm{HCl}(600 \mathrm{~mL})$, and extracted with $\mathrm{CHCl}_{3}(3 \times 500 \mathrm{~mL})$. The combined organic layers were extracted with aqueous $1 \mathrm{M} \mathrm{NaOH}(2 \times 500 \mathrm{~mL})$. The aqueous phase was reacidified with concentrated $\mathrm{HCl}$ and extracted with $\mathrm{CHCl}_{3}(3 \times 500 \mathrm{~mL})$. The combined organic phases were dried and concentrated in vacuo to afford 31a $(7.80 \mathrm{~g}, 68 \%)$ as an orange solid. Characterization data was in agreement with previously reported values. ${ }^{1} \mathrm{New}$ data: ${ }^{1} \mathrm{H}$ NMR $\left(400 \mathrm{MHz}\right.$, acetone- $\left.d_{6}\right): \delta 9.70(\mathrm{br} \mathrm{s}, 1 \mathrm{H}), 8.00(\mathrm{~d}, J=8.6 \mathrm{~Hz}, 1 \mathrm{H}), 7.52(\mathrm{~d}$, $J=2.6 \mathrm{~Hz}, 1 \mathrm{H}), 7.38(\mathrm{dd}, J=2.6,8.6 \mathrm{~Hz}, 1 \mathrm{H}), 6.18(\mathrm{~s}, 1 \mathrm{H}), 4.02(\mathrm{~s}, 3 \mathrm{H}) ;{ }^{13} \mathrm{C}$ NMR $\left(100 \mathrm{MHz}, \mathrm{DMSO}-d_{6}\right) \delta 184.5,181.6$, $163.3,159.6,132.7,128.2,125.6,120.4,111.3,110.3,56.3$.<smiles>COc1ccc2c(c1)C(=O)C=C(O)C2=O</smiles>

31b

2-Hydroxy-6-methoxy-[1,4]naphthoquinone (31b). Oxygen was passed through a solution of $\mathrm{KO} t \mathrm{Bu}(50 \mathrm{~g}, 446 \mathrm{mmol})$ in $\mathrm{HO} t \mathrm{Bu}(500 \mathrm{~mL})$ for $30 \mathrm{~min}$. 6-Methoxytetralone $(\mathbf{3 0 b})(10.0 \mathrm{~g}, 56.8 \mathrm{mmol})$ was added, and oxygen continued to bubble through the reaction mixture for $4 \mathrm{~h}$. The mixture was cooled to $0{ }^{\circ} \mathrm{C}$, quenched with $1 \mathrm{M} \mathrm{HCl}(600 \mathrm{~mL})$, and extracted with $\mathrm{CHCl}_{3}(3 \times 500 \mathrm{~mL})$. The combined organic layers were extracted with aqueous $1 \mathrm{M} \mathrm{NaOH}(2 \times 500 \mathrm{~mL})$. The aqueous phase was reacidified with concentrated $\mathrm{HCl}$ and extracted with $\mathrm{CHCl}_{3}(3 \times 500 \mathrm{~mL})$. The combined organic phases were dried and concentrated in vacuo to afford $31 \mathrm{~b}(7.12 \mathrm{~g}, 62 \%)$ as a yellow solid. Characterization data was in agreement with previously reported values. ${ }^{1}$ New data: ${ }^{1} \mathrm{H}$ NMR $\left(400 \mathrm{MHz}\right.$, acetone- $\left.d_{6}\right): \delta 8.01(\mathrm{~d}, J=8.6 \mathrm{~Hz}, 1 \mathrm{H}), 7.46(\mathrm{~d}, J=2.6 \mathrm{~Hz}, 1 \mathrm{H})$, $7.28(\mathrm{dd}, J=2.6,8.6 \mathrm{~Hz}, 1 \mathrm{H}), 6.17(\mathrm{~s}, 1 \mathrm{H}), 3.98(\mathrm{~s}, 3 \mathrm{H}) ;{ }^{13} \mathrm{C}$ NMR $\left(100 \mathrm{MHz}, \mathrm{DMSO}-d_{6}\right) \delta 184.7,180.3,164.5,160.3$, 134.6, 129.1, 124.0, 119.1, 110.9, 110.1, 56.3.

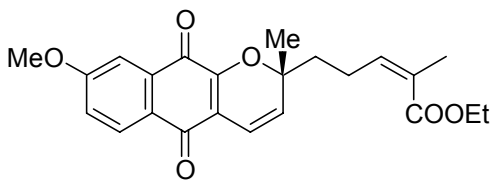

32a

(Z)-5-(8-Methoxy-2-methyl-5,10-dioxo-5,10-dihydro-2 $H$-benzo[g]chromen-2-yl)-2-methyl-pent-2-enoic acid ethyl ester (32a). A solution of naphthoquinone 31a $(728 \mathrm{mg}, 3.57 \mathrm{mmol})$, aldehyde 24 (500 mg, $2.38 \mathrm{mmol})$, $\beta$-alanine (21 mg, $0.24 \mathrm{mmol})$, and HOAc $(0.5 \mathrm{~mL})$ in $\mathrm{PhH}(60 \mathrm{~mL})$ was heated to reflux. After $3 \mathrm{~h}$, the reaction mixture was concentrated. Flash chromatography (2:1 hexanes : EtOAc) afforded chromene 32a $(472 \mathrm{mg}, 50 \%)$ as a red oil: $\mathrm{R}_{\mathrm{f}} 0.43(1: 1$ hexanes : EtOAc); IR: $3045,2979,2919,1711,1677,1648,1598 \mathrm{~cm}^{-1} ;{ }^{1} \mathrm{H}$ NMR $(400 \mathrm{MHz}): \delta 7.96$ (d, $\left.J=8.6 \mathrm{~Hz}, 1 \mathrm{H}\right), 7.46$ (d, $J=$ $2.7 \mathrm{~Hz}, 1 \mathrm{H}), 7.11(\mathrm{dd}, J=2.7,8.6 \mathrm{~Hz}, 1 \mathrm{H}), 6.64(\mathrm{~d}, J=9.9 \mathrm{~Hz}, 1 \mathrm{H}), 5.89$ (t, $J=7.5 \mathrm{~Hz}, 1 \mathrm{H}), 5.64$ (d, $J=9.9 \mathrm{~Hz}, 1 \mathrm{H}), 4.08$ $(\mathrm{q}, J=7.2 \mathrm{~Hz}, 2 \mathrm{H}), 3.88(\mathrm{~s}, 3 \mathrm{H}), 2.57(\mathrm{~m}, 2 \mathrm{H}), 1.92-2.03(\mathrm{~m}, 1 \mathrm{H}), 1.71-1.81(\mathrm{~m}, 4 \mathrm{H}), 1.48(\mathrm{~s}, 3 \mathrm{H}), 1.19(\mathrm{t}, J=7.2 \mathrm{~Hz}, 3 \mathrm{H})$; ${ }^{13} \mathrm{C}$ NMR $(125 \mathrm{MHz}) \delta 181.0,179.6,167.6,163.5,152.2,141.5,133.3,129.4,128.4,127.8,124.7,119.8,117.3,116.3$, 109.9, 82.6, 60.0, 55.8, 40.8, 27.4, 24.4, 20.5, 14.4; HRMS (EI+) Calcd for $\mathrm{C}_{23} \mathrm{H}_{24} \mathrm{O}_{6}\left(\mathrm{M}^{+}\right): 396.1572$, Found: 396.1569 . 
<smiles>CCOC(=O)/C(C)=C\CCC1(C)C=CC2=C(O1)C(=O)c1ccc(OC)cc1C2=O</smiles>

32b

(Z)-5-(7-Methoxy-2-methyl-5,10-dioxo-5,10-dihydro-2H-benzo[g]chromen-2-yl)-2-methyl-pent-2-enoic acid ethyl ester (32b). A solution of naphthoquinone 31a (2.96 g, $14.5 \mathrm{mmol})$, aldehyde 24 (1.017 g, $4.836 \mathrm{mmol})$, $\beta$-alanine (64 mg, $0.73 \mathrm{mmol})$, and HOAc $(0.4 \mathrm{~mL})$ in $\mathrm{PhH}(150 \mathrm{~mL})$ was heated to reflux. After $3 \mathrm{~h}$, the reaction mixture was concentrated. Flash chromatography $\left(2: 1\right.$ hexanes : EtOAc) afforded chromene 32a $(2.98 \mathrm{~g}, 52 \%)$ as an orange solid: mp $80-81{ }^{\circ} \mathrm{C}$; $\mathrm{R}_{\mathrm{f}}$ 0.44 (1:1 hexanes : EtOAc); IR: 3055, 2982, 2932, 1707, 1669, 1651, $1596 \mathrm{~cm}^{-1} ;{ }^{1} \mathrm{H} \mathrm{NMR}(500 \mathrm{MHz}): \delta 8.02(\mathrm{~d}, J=8.5 \mathrm{~Hz}$, $1 \mathrm{H}), 7.53(\mathrm{~d}, J=2.8 \mathrm{~Hz}, 1 \mathrm{H}), 7.11(\mathrm{dd}, J=2.8,8.5 \mathrm{~Hz}, 1 \mathrm{H}), 6.68(\mathrm{~d}, J=10.1 \mathrm{~Hz}, 1 \mathrm{H}), 5.93(\mathrm{~m}, 1 \mathrm{H}), 5.64(\mathrm{~d}, J=10.1 \mathrm{~Hz}$, $1 \mathrm{H}), 4.12(\mathrm{~m}, 2 \mathrm{H}), 3.93(\mathrm{~s}, 3 \mathrm{H}), 2.61(\mathrm{~m}, 2 \mathrm{H}), 1.99-2.07(\mathrm{~m}, 1 \mathrm{H}), 1.82(\mathrm{~m}, 3 \mathrm{H}), 1.75-1.81(\mathrm{~m}, 1 \mathrm{H}), 1.52(\mathrm{~s}, 3 \mathrm{H}), 1.23(\mathrm{t}, J=$ 7.2 Hz, 3H); ${ }^{13} \mathrm{C}$ NMR $(125 \mathrm{MHz}) \delta 181.7,178.6,167.8,164.3,153.0,141.6,133.8,128.8,128.7,127.9,124.9,119.2$, 117.1, 116.3, 110.1, 83.0, 60.1, 55.9, 41.0, 27.6, 24.5, 20.6, 14.2; HRMS (EI+) Calcd for $\mathrm{C}_{23} \mathrm{H}_{24} \mathrm{O}_{6}\left(\mathrm{M}^{+}\right): 396.1572, \mathrm{Found}$ : 396.1570 .

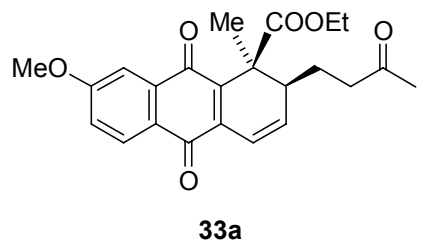

7-Methoxy-1-methyl-9,10-dioxo-2-(3-oxo-butyl)-1,2,9,10-tetrahydro-anthracene-1-carboxylic acid ethyl ester (33a). A solution of chromene 32a (312 $\mathrm{mg}, 0.787 \mathrm{mmol})$ in $\mathrm{PhH}$ was heated to $160{ }^{\circ} \mathrm{C}$ in a sealed tube. After $12 \mathrm{~h}$, the reaction mixture was concentrated and purified by flash chromatography (3:1 then 2:1 hexanes : EtOAc) to afford 33a (252 mg, 81 \%) as a yellow solid: $\mathrm{R}_{\mathrm{f}} 0.20$ (1:1 hexanes : EtOAc); IR: 3055, 2984, 2938, 1726, 1660, $1598,1582 \mathrm{~cm}^{-1}$; ${ }^{1} \mathrm{H} \mathrm{NMR}(400$ $\mathrm{MHz}): \delta 8.04(\mathrm{~d}, J=8.7 \mathrm{~Hz}, 1 \mathrm{H}), 7.46(\mathrm{~d}, J=2.6 \mathrm{~Hz}, 1 \mathrm{H}), 7.16(\mathrm{dd}, J=2.6,8.7 \mathrm{~Hz}, 1 \mathrm{H}), 6.89(\mathrm{~d}, J=9.6 \mathrm{~Hz}, 1 \mathrm{H}), 6.32(\mathrm{dd}$, $J=6.0,9.6 \mathrm{~Hz}, 1 \mathrm{H}), 4.20(\mathrm{~m}, 2 \mathrm{H}), 3.93(\mathrm{~s}, 3 \mathrm{H}), 2.47-2.54(\mathrm{~m}, 2 \mathrm{H}), 2.37(\mathrm{~m}, 1 \mathrm{H}), 2.06(\mathrm{~s}, 3 \mathrm{H}), 1.23(\mathrm{~m}, 3 \mathrm{H}) ;{ }^{13} \mathrm{C} \mathrm{NMR}(125$ $\mathrm{MHz}) \delta$; HRMS (FAB+) Calcd for $\mathrm{C}_{23} \mathrm{H}_{25} \mathrm{O}_{6}(\mathrm{M}+\mathrm{H})^{+}: 397.1651$, Found: 397.1643.

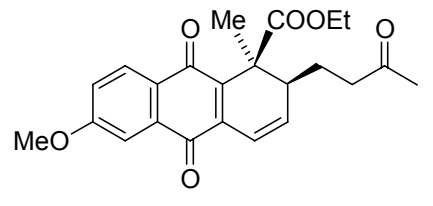

$33 b$

6-Methoxy-1-methyl-9,10-dioxo-2-(3-oxo-butyl)-1,2,9,10-tetrahydro-anthracene-1-carboxylic acid ethyl ester (33b). A solution of chromene $32 \mathrm{~b}(50 \mathrm{mg}, 0.136 \mathrm{mmol})$ in $\mathrm{PhH}$ was heated to $160{ }^{\circ} \mathrm{C}$ in a sealed tube. After $12 \mathrm{~h}$, the reaction mixture was concentrated and purified by flash chromatography (3:1 then 2:1 hexanes : EtOAc) to afford 33b (42 mg, $82 \%)$ as a yellow solid: $\mathrm{mp} 124-125^{\circ} \mathrm{C}$; $\mathrm{R}_{\mathrm{f}} 0.22$ (1:1 hexanes : EtOAc); IR: 2956, 2928, 2855, 1730, $1660,1597 \mathrm{~cm}^{-1} ;{ }^{1} \mathrm{H} \mathrm{NMR}$ $(500 \mathrm{MHz}): \delta 7.98(\mathrm{~d}, J=8.7 \mathrm{~Hz}, 1 \mathrm{H}), 7.54(\mathrm{~d}, J=2.8 \mathrm{~Hz}, 1 \mathrm{H}), 7.19(\mathrm{dd}, J=2.8,8.7 \mathrm{~Hz}, 1 \mathrm{H}), 6.88(\mathrm{~d}, J=9.9 \mathrm{~Hz}, 1 \mathrm{H})$, $6.30(\mathrm{dd}, J=6.0,9.8 \mathrm{~Hz}, 1 \mathrm{H}), 4.20(\mathrm{~m}, 2 \mathrm{H}), 3.95(\mathrm{~s}, 3 \mathrm{H}), 2.47-2.57(\mathrm{~m}, 2 \mathrm{H}), 2.36(\mathrm{~m}, 1 \mathrm{H}), 2.07(\mathrm{~s}, 3 \mathrm{H}), 1.72(\mathrm{~m}, 1 \mathrm{H}), 1.59-$ $1.67(\mathrm{~m}, 1 \mathrm{H}), 1.46(\mathrm{~s}, 3 \mathrm{H}), 1.21(\mathrm{t}, J=7.3 \mathrm{~Hz}, 3 \mathrm{H}) ;{ }^{13} \mathrm{C} \mathrm{NMR}(125 \mathrm{MHz}) \delta 208.0,183.2,182.7,173.8,163.8,142.5,135.8$, 135.4, 133.4, 128.7, 126.6, 120.4, 118.5, 109.7, 60.9, 55.9, 47.4, 43.2, 39.7, 29.9, 26.7, 23.1, 14.0; HRMS (FAB+) Calcd for $\mathrm{C}_{23} \mathrm{H}_{25} \mathrm{O}_{6}(\mathrm{M}+\mathrm{H})^{+}: 397.1651$, Found: 397.1640 .<smiles></smiles>

(Z)-2-(5-Hydroxy-4-methyl-pent-3-enyl)-7-methoxy-2-methyl-2H-benzo[g]chromene-5,10-dione (34). To a solution of 32b (100 mg, $0.252 \mathrm{mmol})$ in $\mathrm{CH}_{2} \mathrm{Cl}_{2}(5 \mathrm{~mL})$ at $-78^{\circ} \mathrm{C}$ was added DIBAL $(0.34 \mathrm{~mL}, 0.51 \mathrm{mmol}, 1.5 \mathrm{M}$ in PhMe). After 1.5 $\mathrm{h}$, the reaction was allowed to warm to $\mathrm{rt}$, quenched with Rochelle's solution $(20 \mathrm{~mL})$, and stirred for $3 \mathrm{~h}$. The reaction mixture was then extracted with $\mathrm{CH}_{2} \mathrm{Cl}_{2}(3 \times 20 \mathrm{~mL})$, and the combined organic layers were dried and concentrated in vасио. Flash chromatography (3:1 then 1:1 hexanes : EtOAc) afforded unreacted $\mathbf{3 2 b}(35 \mathrm{mg}, 35 \%)$ and allylic alcohol 34 $(15 \mathrm{mg}, 17 \%)$ as an orange oil: $\mathrm{R}_{\mathrm{f}} 0.24$ (1:1 hexanes : EtOAc); IR: 3475, 2966, 2928, 2853, 1666, 1650, $1594,1569 \mathrm{~cm}^{-1}$; ${ }^{1} \mathrm{H}$ NMR $(500 \mathrm{MHz}): \delta 8.03(\mathrm{~d}, J=8.5 \mathrm{~Hz}, 1 \mathrm{H}), 7.53(\mathrm{~d}, J=2.6 \mathrm{~Hz}, 1 \mathrm{H}), 7.12(\mathrm{dd}, J=2.6,8.5 \mathrm{~Hz}, 1 \mathrm{H}), 6.68(\mathrm{~d}, J=10.1$ $\mathrm{Hz}, 1 \mathrm{H}), 5.62(\mathrm{~d}, J=10.1 \mathrm{~Hz}, 1 \mathrm{H}), 5.26(\mathrm{t}, J=7.0 \mathrm{~Hz}, 1 \mathrm{H}), 4.11(\mathrm{~d}, J=11.9 \mathrm{~Hz}, 1 \mathrm{H}), 4.07(\mathrm{~d}, J=11.9 \mathrm{~Hz}, 1 \mathrm{H}), 3.94(\mathrm{~s}$, 
3H), $2.21(\mathrm{~m}, 2 \mathrm{H}), 1.95(\mathrm{~m}, 1 \mathrm{H}), 1.67-1.75(\mathrm{~m}, 4 \mathrm{H}), 1.51(\mathrm{~s}, 3 \mathrm{H}) ;{ }^{13} \mathrm{C}$ NMR $(125 \mathrm{MHz}) \delta 181.7,178.7,164.4,153.0,135.1$, 133.8, 129.0, 128.8, 127.2, 124.9, 119.2, 117.1, 116.2, 110.1, 83.0, 61.5, 55.9, 41.6, 27.5, 22.3, 21.3; HRMS (EI+) Calcd for $\mathrm{C}_{21} \mathrm{H}_{22} \mathrm{O}_{5}\left(\mathrm{M}^{+}\right)$: 354.1467 , Found: 354.1467 .

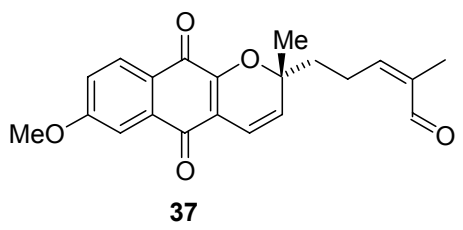

5-(7-Methoxy-2-methyl-5,10-dioxo-5,10-dihydro-2 $\mathrm{H}$-benzo $[\mathrm{g}]$ chromen-2-yl)-2-methyl-pent-2-enal (37). To a solution of oxalyl chloride $\left(0.13 \mathrm{~mL}, 0.26 \mathrm{mmol}, 2.0 \mathrm{M}\right.$ in $\left.\mathrm{CH}_{2} \mathrm{Cl}_{2}\right)$ in $\mathrm{CH}_{2} \mathrm{Cl}_{2}(10 \mathrm{~mL})$ at $-78{ }^{\circ} \mathrm{C}$ was added DMSO $(34 \mu \mathrm{L}, 0.52$ mmol). After $30 \mathrm{~min}$ a solution of alcohol $34(46 \mathrm{mg}, 130 \mathrm{mmol})$ in $\mathrm{CH}_{2} \mathrm{Cl}_{2}(3 \mathrm{~mL})$ was added dropwise. After $45 \mathrm{~min}^{\mathrm{Et}} \mathrm{t}_{3} \mathrm{~N}$ $(0.11 \mathrm{~mL}, 0.78 \mathrm{mmol})$ was added, and the reaction mixture was maintained at $-78{ }^{\circ} \mathrm{C}$ for an additional $45 \mathrm{~min}$. The reaction was then quenched with aqueous $\mathrm{NH}_{4} \mathrm{Cl}(10 \mathrm{~mL})$ and allowed to warm to rt. The mixture was then extracted with $\mathrm{CH}_{2} \mathrm{Cl}_{2}(3$ $\times 10 \mathrm{~mL}$ ), and the combined organic layers were washed with brine $(1 \times 30 \mathrm{~mL})$, dried, and concentrated in vacuo. Flash chromatography afforded aldehyde $37\left(26 \mathrm{mg}, 57 \%\right.$ ) as an orange oil: $\mathrm{R}_{\mathrm{f}} 0.39$ (1:1 hexanes : EtOAc); IR: 2969, 2944, 2919, 2844, 1670, 1650, 1594, $1569 \mathrm{~cm}^{-1}$; ${ }^{1} \mathrm{H}$ NMR (400 MHz): $\delta 10.09$ (s, $\left.1 \mathrm{H}\right), 8.03(\mathrm{~d}, J=8.7 \mathrm{~Hz}, 1 \mathrm{H}), 7.53(\mathrm{~d}, J=2.7 \mathrm{~Hz}, 1 \mathrm{H})$, $7.12(\mathrm{dd}, J=2.7,8.7 \mathrm{~Hz}, 1 \mathrm{H}), 6.73(\mathrm{~d}, J=10.2 \mathrm{~Hz}, 1 \mathrm{H}), 6.49(\mathrm{t}, J=8.07 \mathrm{~Hz}, 1 \mathrm{H}), 5.62(\mathrm{~d}, J=10.2 \mathrm{~Hz}, 1 \mathrm{H}), 3.94(\mathrm{~s}, 3 \mathrm{H})$, $2.74(\mathrm{~m}, 2 \mathrm{H}), 2.05-2.16(\mathrm{~m}, 1 \mathrm{H}), 1.77-1.89(\mathrm{~s}, 1 \mathrm{H}), 1.71(\mathrm{~s}, 3 \mathrm{H}), 1.54(\mathrm{~s}, 3 \mathrm{H}) ;{ }^{13} \mathrm{C}$ NMR $(100 \mathrm{MHz}) \delta 190.7,181.5,178.5$, $164.4,152.8,147.8,136.3,133.7,128.2,124.8,119.3,116.9,116.8,110.2,82.6,55.9,41.3,27.6,21.8,16.4$; HRMS (EI+) Calcd for $\mathrm{C}_{21} \mathrm{H}_{20} \mathrm{O}_{5}\left(\mathrm{M}^{+}\right)$: 352.1311, Found: 352.1308.

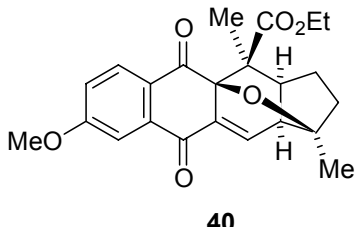

Isopinnatal analog 40. A solution of 32b $(21 \mathrm{mg}, 0.053 \mathrm{mmol})$ in $\mathrm{CH}_{2} \mathrm{Cl}_{2}(1 \mathrm{~mL})$ was subjected to $14 \mathrm{kbar}$. After $50 \mathrm{~h}$, the reaction mixture was concentrated in vacuo. Flash chromatography (2:1 hexanes : EtOAc) afforded $40(11 \mathrm{mg}, 52 \%)$ as a pale yellow solid: $\mathrm{mp}: 124-125^{\circ} \mathrm{C}$; $\mathrm{R}_{\mathrm{f}} 0.39$ (1:1 hexanes : EtOAc); IR: 2976, 2934, 2854, 1736, 1688, 1670, 1617, $1593 \mathrm{~cm}^{-}$ ${ }^{1} ;{ }^{1} \mathrm{H}$ NMR $(500 \mathrm{MHz}): \delta 8.03(\mathrm{~d}, J=8.7 \mathrm{~Hz}, 1 \mathrm{H}), 7.80(\mathrm{~d}, J=7.1 \mathrm{~Hz}, 1 \mathrm{H}), 7.60(\mathrm{~d}, J=2.6 \mathrm{~Hz}, 1 \mathrm{H}), 7.21(\mathrm{dd}, J=2.6,8.7$ $\mathrm{Hz}, 1 \mathrm{H}), 4.27(\mathrm{dq}, J=7.2,10.8 \mathrm{~Hz}, 1 \mathrm{H}), 4.19(\mathrm{dq}, J=7.2,10.8 \mathrm{~Hz}, 1 \mathrm{H}), 3.95(\mathrm{~s}, 3 \mathrm{H}), 2.88(\mathrm{dd}, J=4.1,7.1 \mathrm{~Hz}, 1 \mathrm{H}), 2.50-$ $2.56(\mathrm{~m}, 1 \mathrm{H}), 2.03-2.10(\mathrm{~m}, 1 \mathrm{H}), 1.89-1.93(\mathrm{~m}, 1 \mathrm{H}), 1.75-1.82(\mathrm{~m}, 2 \mathrm{H}), 1.32(\mathrm{t}, J=7.2 \mathrm{~Hz}, 3 \mathrm{H}), 1.13(\mathrm{~s}, 3 \mathrm{H}), 0.84(\mathrm{~s}, 3 \mathrm{H})$; ${ }^{13} \mathrm{C}$ NMR $(125 \mathrm{MHz}) \delta 190.5,179.4,171.9,164.5,142.4,141.2,136.9,130.8,130.2,121.9,109.3,84.9,78.4,60.9,56.3$, 56.0, 50.4, 47.1, 37.2, 27.8, 24.5, 24.4, 14.1; HRMS (EI+) Calcd for $\mathrm{C}_{23} \mathrm{H}_{24} \mathrm{O}_{6}\left(\mathrm{M}^{+}\right): 396.1572$, Found: 396.1572.

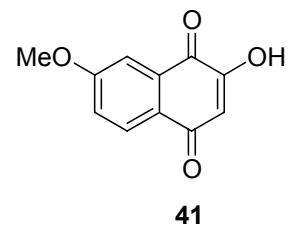

2,7-Dihydroxy-[1,4]naphthoquinone (41). To a melt of $\mathrm{AlCl}_{3}(30.0 \mathrm{~g}, 227.5 \mathrm{mmol})$ and $\mathrm{NaCl}(7.00 \mathrm{~g}, 119.8 \mathrm{mmol})$ at 180 ${ }^{\circ} \mathrm{C}$ was added finely ground naphthoquinone $31 \mathrm{a}(5.00 \mathrm{~g}, 24.5 \mathrm{mmol})$. After $5 \mathrm{~min}$, the heat was removed and the reaction mixture allowed to cool for $5 \mathrm{~min}$. The mixture was then carefully poured into a slurry of ice $(750 \mathrm{~mL})$ in concentrated $\mathrm{HCl}$ $(200 \mathrm{~mL})$. The mixture was extracted with $\mathrm{Et}_{2} \mathrm{O}(3 \times 500 \mathrm{ml})$, and the combined organic phases were concentrated in vacuo to afford the demethylated naphthoquinone $41(2.47 \mathrm{~g}, 53 \%)$ as a yellow solid. Spectral data agreed with previously reported values. ${ }^{2}$

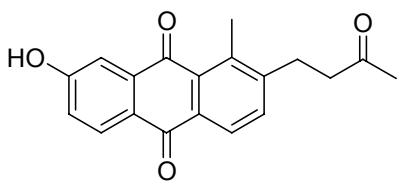

anthrakunthone (14)

Anthrakunthone (14). To a solution of sterekunthal A (13) $(36 \mathrm{mg}, 0.105 \mathrm{mmol})$ in MeCN (10 mL) was added Wilkinson's catalyst $\left(\mathrm{Rh}\left[\mathrm{PPh}_{3}\right]_{3} \mathrm{Cl}\right)(120 \mathrm{mg}, 0.130 \mathrm{mmol})$, and the reaction mixture was heated at reflux $4 \mathrm{~h}$. The mixture was allowed to cool, filtered through Celite, and concentrated in vacuo. Flash chromatography (2:1 hexanes : EtOAc) afforded anthrakunthone (14.8 mg, $47 \%$ ) as a yellow solid. New data: ${ }^{13} \mathrm{C}$ NMR $\left(100 \mathrm{MHz}\right.$, acetone- $\left.d_{6}\right) \delta 206.8,186.2,182.3,163.7$, 
163.5, 148.7, 140.4, 138.5, 135.2, 134.4, 132.9, 129.9, 126.3, 125.8, 121.6, 121.5, 113.3, 43.5, 28.7, 17.5. ${ }^{3}$. Spectral data agreed with published values. ${ }^{4}$ See table below for data comparison.

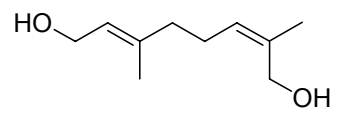

(Z,E)-2,6-Dimethyl-octa-2,6-diene-1,8-diol. To a solution of alcohol $44(1.00 \mathrm{~g}, 2.45 \mathrm{mmol})$ in $\mathrm{THF}(10 \mathrm{~mL})$ at $0{ }^{\circ} \mathrm{C}$ was added TBAF $(3.7 \mathrm{~mL}, 3.7 \mathrm{mmol}, 1 \mathrm{M}$ in THF). After $1 \mathrm{~h}$, the reaction mixture was diluted with brine $(25 \mathrm{~mL})$ and extracted with $\mathrm{Et}_{2} \mathrm{O}(3 \times 25 \mathrm{ml})$. The combined organic phases were washed with aqueous $\mathrm{NaHCO}_{3}(1 \times 50 \mathrm{ml})$ and brine $(1 \times 50 \mathrm{ml})$, dried, and concentrated in vacuo. Flash chromatography (1:2 hexanes : EtOAc) afforded the title diol (382 $\mathrm{mg}, 92 \%)$ as a clear oil: $\mathrm{R}_{\mathrm{f}} 0.14\left(1: 1\right.$ hexanes : EtOAc); IR: 3329, 2964, 2920, $2877 \mathrm{~cm}^{-1} ;{ }^{1} \mathrm{H}$ NMR $(500 \mathrm{MHz}): \delta 5.38(\mathrm{t}, J=7.0 \mathrm{~Hz}, 1 \mathrm{H})$; $5.25(\mathrm{t}, J=7.6 \mathrm{~Hz}, 1 \mathrm{H}), 4.13(\mathrm{~d}, J=7.0 \mathrm{~Hz}, 2 \mathrm{H}) ; 4.09(\mathrm{~s}, 2 \mathrm{H}), 2.17(\mathrm{~m}, 2 \mathrm{H}), 2.01(\mathrm{~m}, 2 \mathrm{H}), 1.79(\mathrm{~s}, 3 \mathrm{H}), 1.68(\mathrm{~s}, 3 \mathrm{H}), 1.55$ (br s, 2H); ${ }^{13} \mathrm{C}$ NMR $(125 \mathrm{MHz}) \delta 139.2,134.9,127.3,124.0,61.4,59.1,39.2,25.8,21.1,16.4$; HRMS (FAB + ) Calcd for $\mathrm{C}_{10} \mathrm{H}_{18} \mathrm{LiO}_{2}(\mathrm{M}+\mathrm{Li})^{+}:$177.1467, Found: 177.1472 .

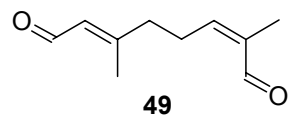

(Z,E)-2,6-Dimethyl-octa-2,6-dienedial (49). The Dess-Martin periodinane (600 $\mathrm{mg}, 1.41 \mathrm{mmol})$ was added to a solution of the above diol $(100 \mathrm{mg}, 0.587 \mathrm{mmol})$ in $\mathrm{CH}_{2} \mathrm{Cl}_{2}(6 \mathrm{~mL})$. After $1 \mathrm{~h}$ the reaction mixture was quenched with an aqueous solution of $\mathrm{NaHCO}_{3}$ and $\mathrm{Na}_{2} \mathrm{~S}_{2} \mathrm{O}_{3}\left(20 \mathrm{~mL}, 1: 1: 1 \mathrm{v} / \mathrm{v} / \mathrm{v}\right.$ sat. $\mathrm{NaHCO}_{3}$ : sat. $\left.\mathrm{Na}_{2} \mathrm{~S}_{2} \mathrm{O}_{3}: \mathrm{H}_{2} \mathrm{O}\right)$, then extracted with $\mathrm{CH}_{2} \mathrm{Cl}_{2}(3 \times$ $20 \mathrm{ml})$. The combined organic phases were dried and concentrated in vacuo. Flash chromatography (2:1 hexanes : EtOAc) afforded $49(80 \mathrm{mg}, 83 \%)$ as a clear oil: $\mathrm{R}_{\mathrm{f}} 0.37\left(1: 1\right.$ hexanes : EtOAc); IR: 2951, 2924, 2857, 2778, $1673 \mathrm{~cm}^{-1} ;{ }^{1} \mathrm{H}$ NMR (500 MHz): $\delta 10.13(\mathrm{~s}, 1 \mathrm{H}), 10.00(\mathrm{~d}, J=7.9 \mathrm{~Hz}, 1 \mathrm{H}), 6.43(\mathrm{~m}, 1 \mathrm{H}), 5.89(\mathrm{~d}, J=7.8 \mathrm{~Hz}, 1 \mathrm{H}), 2.80(\mathrm{~m}, 2 \mathrm{H}), 2.39(\mathrm{~m}, 2 \mathrm{H})$, $2.19(\mathrm{~d}, J=1.1 \mathrm{~Hz}, 3 \mathrm{H}), 1.77(\mathrm{~s}, 3 \mathrm{H}) ;{ }^{13} \mathrm{C}$ NMR $(125 \mathrm{MHz}) \delta 190.9,190.5,161.0,146.3,137.0,127.9,40.0,24.1,17.5$, 16.5; HRMS (FAB+) Calcd for $\mathrm{C}_{10} \mathrm{H}_{15} \mathrm{O}_{2}(\mathrm{M}+\mathrm{H})^{+}:$167.1072, Found: 167.1071 .<smiles>O=C1C=C(O)C(=O)c2cc(O)ccc21</smiles>

50

2,6-Dihydroxy-[1,4]naphthoquinone (50). To a melt of $(30.0 \mathrm{~g}, 227.5 \mathrm{mmol})$ and $\mathrm{NaCl}(7.00 \mathrm{~g}, 119.8 \mathrm{mmol})$ at $180{ }^{\circ} \mathrm{C}$ was added finely ground naphthoquinone $31 \mathrm{a}(5.00 \mathrm{~g}, 24.5 \mathrm{mmol})$. After $5 \mathrm{~min}$, the heat was removed and the reaction mixture allowed to cool for $5 \mathrm{~min}$. The mixture was then carefully poured into a slurry of ice $(750 \mathrm{~mL})$ in concentrated $\mathrm{HCl}$ $(200 \mathrm{~mL})$. The mixture was extracted with $\mathrm{Et}_{2} \mathrm{O}(3 \times 500 \mathrm{ml})$, and the combined organic phases were concentrated in vacuo to afford the demethylated naphthoquinone $41(2.28 \mathrm{~g}, 49 \%)$ as a yellow solid. Spectral data agreed with previously reported values. ${ }^{2}$

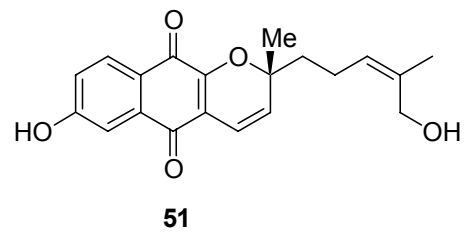

(Z)-7-Hydroxy-2-(5-hydroxy-4-methyl-pent-3-enyl)-2-methyl-2H-benzo[g]chromene-5,10-dione (51). A solution of unsaturated aldehyde 46 (695.9 mg, $2.758 \mathrm{mmol})$, naphthoquinone 50 (1.001g, $5.30 \mathrm{mmol})$, EDDA (126 mg, $0.699 \mathrm{mmol})$, and $\mathrm{Na}_{2} \mathrm{SO}_{4}(4.054 \mathrm{~g}, 28.54 \mathrm{mmol})$ in THF $(100 \mathrm{ml})$ was stirred for $4 \mathrm{~h}$ at rt. The reaction mixture was filtered then diluted with $\mathrm{NH}_{4} \mathrm{Cl}(100 \mathrm{ml})$ and extracted with $\mathrm{Et}_{2} \mathrm{O}(5 \times 50 \mathrm{ml})$. The combined organic phases were washed with brine $(250 \mathrm{ml})$, dried, and concentrated in vacuo. Flash chromatography $\left(1: 1\right.$ pentane : $\left.\mathrm{Et}_{2} \mathrm{O}\right)$ afforded the protected chromene as a mixture of diastereomers $(965.2 \mathrm{mg}, 82 \%)$ as an orange oil which slowly solidified: ${ }^{1} \mathrm{H}$ NMR (400 MHz): $\delta 8.06(\mathrm{br} \mathrm{s}, 1 \mathrm{H}), 7.96(\mathrm{~d}$, $J=8.6 \mathrm{~Hz}, 1 \mathrm{H}), 7.52(\mathrm{~d}, J=2.5 \mathrm{~Hz}, 1 \mathrm{H}), 7.07(\mathrm{dd}, J=2.5,8.6 \mathrm{~Hz}, 1 \mathrm{H}), 6.62(\mathrm{~d}, J=10.1 \mathrm{~Hz}, 1 \mathrm{H}), 5.61(\mathrm{~d}, J=10.1 \mathrm{~Hz}$, $1 \mathrm{H}), 5.33(\mathrm{t}, J=7.1 \mathrm{~Hz}, 1 \mathrm{H}), 4.57(\mathrm{~m}, 1 \mathrm{H}), 3.98-4.14(\mathrm{~m}, 2 \mathrm{H}), 3.85(\mathrm{~m}, 1 \mathrm{H}), 3.50(\mathrm{~m}, 1 \mathrm{H}), 2.21(\mathrm{~m}, 2 \mathrm{H}), 1.87-1.99(\mathrm{~m}, 3 \mathrm{H})$, 1.74-1.84 $(\mathrm{m}, 1 \mathrm{H}), 1.61-1.74(\mathrm{~m}, 4 \mathrm{H}), 1.43-1.61(\mathrm{~m}, 6 \mathrm{H})$. A solution of the above THP-protected compound $(357.4 \mathrm{mg}$, $0.842 \mathrm{mmol})$ and $\mathrm{TsOH} \cdot \mathrm{H}_{2} \mathrm{O}(26.8 \mathrm{mg}, 0.150 \mathrm{mmol})$ in $\mathrm{MeOH}(22 \mathrm{ml})$ was stirred for $2.5 \mathrm{~h}$ at rt. The reaction mixture was diluted with $\mathrm{H}_{2} \mathrm{O}(50 \mathrm{ml})$ and extracted with diethyl ether $(5 \times 30 \mathrm{~mL})$. The combined organic phases were washed with brine $(30 \mathrm{ml})$, dried, and concentrated in vacuo. Flash chromatography $(2: 1$ hexanes : EtOAc, then 1:1 hexanes : EtOAc) afforded allylic alcohol $51(272.9 \mathrm{mg}, 95 \%)$ as a red oil: $\mathrm{R}_{\mathrm{f}} 0.16$ (1:1 hexanes : EtOAc); IR: 3268, 2970, 2926, 2883, 1649, $1582 \mathrm{~cm}^{-1} ;{ }^{1} \mathrm{H}$ NMR $(500 \mathrm{MHz}): \delta 8.79(\mathrm{br} \mathrm{s}, 1 \mathrm{H}), 7.88(\mathrm{~d}, J=8.3 \mathrm{~Hz}, 1 \mathrm{H}), 7.43(\mathrm{~d}, J=2.5 \mathrm{~Hz}, 1 \mathrm{H}), 7.03(\mathrm{dd}, J=2.5,8.3$ $\mathrm{Hz}, 1 \mathrm{H}), 6.57(\mathrm{~d}, J=10.1 \mathrm{~Hz}, 1 \mathrm{H}), 5.59(\mathrm{~d}, J=10.1 \mathrm{~Hz}, 1 \mathrm{H}), 5.26(\mathrm{t}, J=7.3 \mathrm{~Hz}, 1 \mathrm{H}), 4.13(\mathrm{~m}, 2 \mathrm{H}), 2.72(\mathrm{br} \mathrm{s}, 1 \mathrm{H}), 2.22$ 
$(\mathrm{m}, 2 \mathrm{H}), 1.83-1.94(\mathrm{~m}, 1 \mathrm{H}), 1.66(\mathrm{~m}, 4 \mathrm{H}), 1.47(\mathrm{~s}, 3 \mathrm{H}) ;{ }^{13} \mathrm{C}$ NMR $(100 \mathrm{MHz}) \delta 182.0,179.0,162.6,153.2,134.5,133.7$,

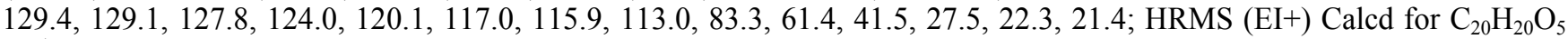
$\left(\mathrm{M}^{+}\right): 340.1311$, Found: 340.1315 .<smiles>C/C(C=O)=C/CCC1(C)C=CC2=C(O1)C(=O)c1ccc(O)cc1C2=O</smiles>

(Z)-5-(7-Hydroxy-2-methyl-5,10-dioxo-5,10-dihydro-2H-benzo[g]chromen-2-yl)-2-methyl-pent-2-enal (52). To a solution of alcohol $51(270.7 \mathrm{mg}, 0.795 \mathrm{mmol})$ in $\mathrm{CH}_{2} \mathrm{Cl}_{2}(35 \mathrm{ml})$ was added the Dess-Martin periodinane (418.2 mg, 0.986 mmol). The reaction mixture was stirred for $4.5 \mathrm{~h}$ at rt. The mixture was diluted with $\mathrm{Na}_{2} \mathrm{~S}_{2} \mathrm{O}_{3}(50 \mathrm{~mL})$ and extracted with $\mathrm{CH}_{2} \mathrm{Cl}_{2}(3 \times 50 \mathrm{~mL})$. The combined organic phases were washed with brine $(30 \mathrm{~mL})$, dried, and concentrated in vacuo. Flash chromatography (3:1 hexanes : EtOAc) afforded unsaturated aldehyde $\mathbf{5 2}(228.2 \mathrm{mg}, 85 \%)$ as a red oil: $\mathrm{R}_{\mathrm{f}} 0.38(1: 2 \mathrm{hexanes}$ : EtOAc); IR: 3273, 2975, 2926, 1657, $1581 \mathrm{~cm}^{-1}$; ${ }^{1} \mathrm{H}$ NMR (400 MHz): $\delta 10.07$ (s, 1H), 7.99 (m, $\left.2 \mathrm{H}\right), 7.56(\mathrm{~d}, J=2.5 \mathrm{~Hz}$, $1 \mathrm{H}), 7.12(\mathrm{dd}, J=2.5,8.6 \mathrm{~Hz}, 1 \mathrm{H}), 6.69(\mathrm{~d}, J=10.1 \mathrm{~Hz}, 1 \mathrm{H}), 6.51(\mathrm{t}, J=7.8 \mathrm{~Hz}, 1 \mathrm{H}), 5.62(\mathrm{~d}, J=10.2 \mathrm{~Hz}, 1 \mathrm{H}), 2.75(\mathrm{~m}$, 2H), $2.10(\mathrm{~m}, 1 \mathrm{H}), 1.83(\mathrm{~m}, 1 \mathrm{H}), 1.69(\mathrm{~s}, 3 \mathrm{H}), 1.53(\mathrm{~s}, 3 \mathrm{H}) ;{ }^{13} \mathrm{C}$ NMR $(100 \mathrm{MHz}) \delta 191.3,181.9,178.7,162.2,153.0,148.6$, $136.2,133.8,129.5,128.4,124.3,120.2,116.9,116.6,113.1,82.9,41.3,27.8,21.9,16.4$; $\mathrm{HRMS}(\mathrm{EI}+) \mathrm{Calcd}_{\text {for }} \mathrm{C}_{20} \mathrm{H}_{18} \mathrm{O}_{5}$ $\left(\mathrm{M}^{+}\right): 338.1154$, Found: 338.1154 .

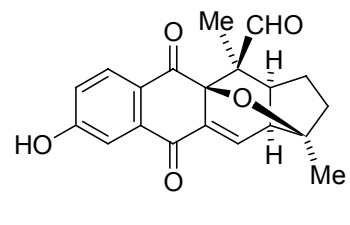

isopinnatal (9)

Isopinnatal (9). Compound $52(130 \mathrm{mg}, 0.385 \mathrm{mmol})$ was let stand at rt for 14 days. Flash chromatography (3:2 hexanes : EtOAc) provided isopinnatal $(105 \mathrm{mg}, 81 \%)$ as a pale yellow solid. Spectroscopic data was in agreement with reported values. ${ }^{5}$ See below for data comparison.

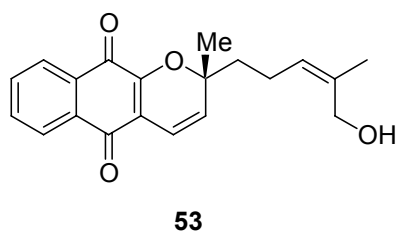

(Z)-2-(5-Hydroxy-4-methyl-pent-3-enyl)-2-methyl-2H-benzo[g]chromene-5,10-dione (53). A solution of unsaturated aldehyde 46 (430.0 mg, $1.70 \mathrm{mmol})$, naphthoquinone 26 (596.3 mg, $3.42 \mathrm{mmol})$, $\beta$-alanine (22.0 mg, $0.246 \mathrm{mmol})$, and HOAc $(0.75 \mathrm{~mL})$ in $\mathrm{PhH}(60 \mathrm{~mL})$ was heated at reflux for $20 \mathrm{~h}$. The reaction mixture was then concentrated in vacuo. Flash chromatography $(3: 1$ hexanes : EtOAc) afforded the protected chromene as a mixture of diastereomers $(600 \mathrm{mg}, 86 \%)$, as a red oil: ${ }^{1} \mathrm{H}$ NMR $(400 \mathrm{MHz}): \delta 7.98-8.03(\mathrm{~m}, 2 \mathrm{H}) 7.58-7.68(\mathrm{~m}, 2 \mathrm{H}), 6.64(\mathrm{~d}, J=10.1 \mathrm{~Hz}, 1 \mathrm{H}), 5.63(\mathrm{~d}, J=10.1 \mathrm{~Hz}, 1 \mathrm{H})$, $5.28(\mathrm{~m}, 1 \mathrm{H}), 4.47(\mathrm{~m}, 1 \mathrm{H}), 3.98(\mathrm{~m}, 2 \mathrm{H}), 3.74(\mathrm{~m}, 1 \mathrm{H}), 3.38(\mathrm{~m}, 1 \mathrm{H}), 2.17(\mathrm{~m}, 2 \mathrm{H}), 1.91(\mathrm{~m}, 1 \mathrm{H}), 1.53-1.78(\mathrm{~m}, 6 \mathrm{H}), 1.35-$ $1.52(\mathrm{~m}, 7 \mathrm{H})$. A solution of the above THP-protected compound (417.0 $\mathrm{mg}, 1.02 \mathrm{mmol})$ and $\mathrm{TsOH} \cdot \mathrm{H}_{2} \mathrm{O}(25.0 \mathrm{mg}, 0.130$ $\mathrm{mmol})$ in $\mathrm{MeOH}(11 \mathrm{ml})$ was stirred for $3 \mathrm{~h}$ at $\mathrm{rt}$. The reaction mixture was diluted with $\mathrm{H}_{2} \mathrm{O}(50 \mathrm{ml})$ and extracted with diethyl ether $(5 \times 30 \mathrm{ml})$. The combined organic phases were washed with $\mathrm{NaHCO}_{3}(30 \mathrm{ml})$ and brine $(30 \mathrm{ml})$, dried, and concentrated in vacuo. Flash chromatography (3:1 hexanes : EtOAc) afforded allylic alcohol $\mathbf{5 3}$ (330 mg, $100 \%)$ as a red oil: $\mathrm{R}_{\mathrm{f}} 0.30$ (1:1 hexanes : EtOAc); IR: 3510, 2971, 2927, 2880, 1673, 1650, 1595, $1571 \mathrm{~cm}^{-1} ;{ }^{1} \mathrm{H} \mathrm{NMR}(400 \mathrm{MHz}): \delta 8.01(\mathrm{~m}$, $2 \mathrm{H}), 7.64(\mathrm{~m}, 2 \mathrm{H}), 6.64(\mathrm{~d}, J=10.1 \mathrm{~Hz}, 1 \mathrm{H}), 5.63(\mathrm{~d}, J=10.1 \mathrm{~Hz}, 1 \mathrm{H}), 5.21(\mathrm{t}, J=7.0 \mathrm{~Hz}, 1 \mathrm{H}), 4.05(\mathrm{~m}, 2 \mathrm{H}), 2.18(\mathrm{~m}, 2 \mathrm{H})$, 2.03 (br s, $1 \mathrm{H}), 1.86-1.95(\mathrm{~m}, 1 \mathrm{H}), 1.70(\mathrm{~m}, 4 \mathrm{H}), 1.47(\mathrm{~s}, 3 \mathrm{H}) ;{ }^{13} \mathrm{C}$ NMR $(100 \mathrm{MHz}) \delta 181.6,179.6,152.5,135.2,133.9$, 133.1, 131.4, 131.3, 129.6, 126.8, 126.1, 126.0, 116.8, 116.0, 82.8, 61.2, 41.5, 27.3, 22.2, 21.2; HRMS (EI+) Calcd for $\mathrm{C}_{20} \mathrm{H}_{20} \mathrm{O}_{4}\left(\mathrm{M}^{+}\right): 324.1362$, Found: 324.1360 .

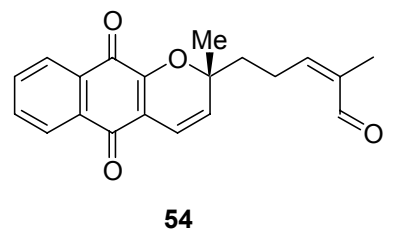

(Z)-2-Methyl-5-(2-methyl-5,10-dioxo-5,10-dihydro-2H-benzo[g]chromen-2-yl)-pent-2-enal (54). To a solution of oxalyl chloride $\left(0.725 \mathrm{ml}, 1.45 \mathrm{mmol}, 2.0 \mathrm{M}\right.$ in $\left.\mathrm{CH}_{2} \mathrm{Cl}_{2}\right)$ in $\mathrm{CH}_{2} \mathrm{Cl}_{2}(20 \mathrm{ml})$ at $-55{ }^{\circ} \mathrm{C}$, DMSO $(0.21 \mathrm{ml}, 2.95 \mathrm{mmol})$ was added. The solution was stirred at $-55{ }^{\circ} \mathrm{C}$ for 5 minutes after which a solution of $\mathbf{5 3}\left(236.5 \mathrm{mg}, 0.729 \mathrm{mmol}^{2}\right.$ in $\mathrm{CH}_{2} \mathrm{Cl}_{2}(20 \mathrm{ml})$ was 
added dropwise. After $1 \mathrm{~h}, \mathrm{Et}_{3} \mathrm{~N}(0.60 \mathrm{ml}, 4.33 \mathrm{mmol})$ was added and the reaction mixture was maintained for $5 \mathrm{~min}$ at -55 ${ }^{\circ} \mathrm{C}$, then allowed to warm to rt. The mixture was diluted with $\mathrm{H}_{2} \mathrm{O}(50 \mathrm{ml})$ and extracted with $\mathrm{CH}_{2} \mathrm{Cl}_{2}(5 \times 20 \mathrm{ml})$. The combined organic phases were washed with $10 \% \mathrm{HCl}(30 \mathrm{ml}), \mathrm{H}_{2} \mathrm{O}(30 \mathrm{ml}), \mathrm{NaHCO}_{3}(30 \mathrm{ml})$, and brine $(30 \mathrm{ml})$, dried, and concentrated in vacuo. Flash chromatography (3:1 hexanes : EtOAc) afforded unsaturated aldehyde $\mathbf{5 4}(178 \mathrm{mg}, \mathbf{7 6} \%)$ as an orange oil: $\mathrm{R}_{\mathrm{f}} 0.46$ (1:1 hexanes : EtOAc); IR: 2976, 2925, 2868, 1668, 1650, 1595, $1571 \mathrm{~cm}^{-1} ;{ }^{1} \mathrm{H}$ NMR (400 MHz): $\delta 9.98$ $(\mathrm{s}, 1 \mathrm{H}), 7.90(\mathrm{~m}, 2 \mathrm{H}), 7.56(\mathrm{~m}, 2 \mathrm{H}), 6.59(\mathrm{~d}, J=10.2 \mathrm{~Hz}, 1 \mathrm{H}), 6.40(\mathrm{t}, J=8.1 \mathrm{~Hz}, 1 \mathrm{H}), 5.61(\mathrm{~d}, J=10.1 \mathrm{~Hz}, 1 \mathrm{H}), 2.65(\mathrm{~m}$, $2 \mathrm{H}), 1.93-2.05(\mathrm{~m}, 1 \mathrm{H}), 1.72-1.83(\mathrm{~m}, 1 \mathrm{H}), 1.57(\mathrm{~s}, 3 \mathrm{H}), 1.44(\mathrm{~s}, 3 \mathrm{H}) ;{ }^{13} \mathrm{C}$ NMR $(100 \mathrm{MHz}) \delta 190.4,181.1,179.0,151.9$, 147.6, 135.8, 133.8, 133.0, 131.0, 130.9, 128.9, 125.9, 125.8, 116.9, 116.3, 82.3, 40.9, 27.2, 21.4, 16.1; HRMS (EI+) Calcd for $\mathrm{C}_{20} \mathrm{H}_{18} \mathrm{O}_{4}\left(\mathrm{M}^{+}\right)$: 322.1205 , Found: 322.1209 .

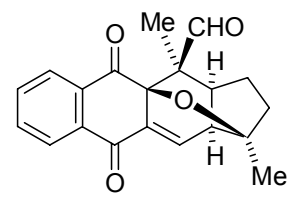

sterekunthal B (10)

Sterekunthal B (10). Aldehyde $54(103.0 \mathrm{mg}, 0.320 \mathrm{mmol})$ was left standing at $\mathrm{rt}$ for 100 days. Flash chromatography (3:1 hexanes : EtOAc) afforded sterekunthal B a pale yellow solid (72.2 mg, 70\%). Recrystalization by layering $\left(\mathrm{CH}_{2} \mathrm{Cl}_{2}\right.$ : hexanes) yielded nearly colorless cubes suitable for X-ray diffraction. New data: ${ }^{13} \mathrm{C}$ NMR $(125 \mathrm{MHz}) \delta 204.5,193.4,179.0$, 143.0, 140.0, 135.5, 135.4, 135.0, 134.6, 128.1, 127.9, 85.0, 78.4, 56.6, 50.7, 47.0, 37.8, 26.1, 24.6, 18.9. Spectroscopic data was in agreement with published values. ${ }^{4}$ See table below for data comparison.
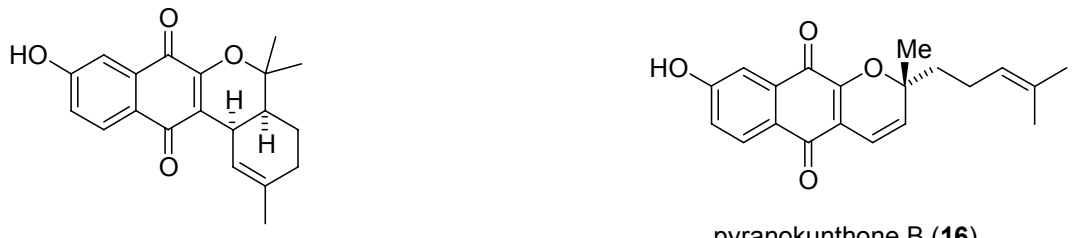

pyranokunthone A (15)

pyranokunthone B (16)

Pyranokunthone A (15) and pyranokunthone B (16). A solution of citral (1.0 mL, $5.76 \mathrm{mmol})$, naphthoquinone 41 (547 $\mathrm{mg}, 2.89 \mathrm{mmol}), \beta$-alanine $(51 \mathrm{mg}, 0.58 \mathrm{mmol})$, and HOAc $(0.2 \mathrm{~mL})$ in $\mathrm{PhH}(30 \mathrm{~mL})$ was heated at reflux for $24 \mathrm{~h}$. The reaction mixture was diluted with $\mathrm{H}_{2} \mathrm{O}(200 \mathrm{ml})$, extracted with EtOAc $(5 \times 100 \mathrm{ml})$, dried, and concentrated in vacuo. Flash chromatography (3:1 then 1:1 hexanes : EtOAc) afforded pyranokunthone B (466 mg, $50 \%$ ) as a red oil, which slowly solidified upon standing at rt. Pyranokunthone A was further purified by reverse phase HPLC $\left(3: 1 \mathrm{MeOH}: \mathrm{H}_{2} \mathrm{O}\right)$ and isolated (44 mg, $5 \%$ ) as a colorless solid. New data for 16: ${ }^{13} \mathrm{C}$ NMR $(125 \mathrm{MHz}) \delta 181.6,180.5,161.2,152.4,133.3,132.3$, $130.1,129.1,124.4,123.4,121.0,117.6,116.0,113.0,83.2,41.5,27.5,25.6,22.7,17.6$. Spectral data agreed with published values. ${ }^{4}$ See table below for data comparison.

Typical procedure for asymmetric pinnatal (8) studies. A solution of $\mathrm{Sc}(\mathrm{OTf})_{3}(4.4 \mathrm{mg}, 0.00894 \mathrm{mmol})$ and $(S, S)$ phenylpybox ( $3.3 \mathrm{mg}, 0.00921 \mathrm{mmol})$ in $\mathrm{CH}_{2} \mathrm{Cl}_{2}(1 \mathrm{~mL})$ was stirred at $\mathrm{rt}$ for $1 \mathrm{~h}$ over $3 \AA$ molecular sieves. A solution of aldehyde 21 (19 mg, $0.0562 \mathrm{mmol})$ in $\mathrm{CH}_{2} \mathrm{Cl}_{2}(1 \mathrm{~mL})$ was added. After $12 \mathrm{~h}$, the reaction mixture was diluted with aqueous $\mathrm{NH}_{4} \mathrm{Cl}(10 \mathrm{~mL})$ and extracted with $\mathrm{CH}_{2} \mathrm{Cl}_{2}(3 \times 10 \mathrm{ml})$. The combined organic extracts were dried and concentrated in vacuo. Flash chromatography (2:1 hexanes : EtOAc, then 1:1 hexanes : EtOAc) afforded pinnatal (8) (7.6 mg, $40 \%)$. Chiral HPLC analysis showed an enantiomeric excess of $55 \%$.

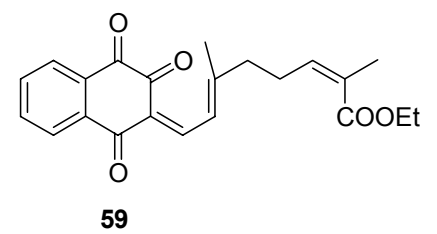

(2,6-Dimethyl-8-(1,3,4-trioxo-3,4-dihydro-1 $H$-naphthalen-2-ylidene)-octa-2,6-dienoic acid ethyl ester (59). To a solution of $27(75 \mathrm{mg}, 0.204 \mathrm{mmol})$ in $\mathrm{CH}_{2} \mathrm{Cl}_{2}(2 \mathrm{~mL})$ at $-78{ }^{\circ} \mathrm{C}$ was added $\mathrm{TiCl}_{4}\left(0.23 \mathrm{~mL}, 0.23 \mathrm{mmol}, 1.0 \mathrm{M}_{10} \mathrm{CH}_{2} \mathrm{Cl}_{2}\right)$. After $2 \mathrm{~h}, \mathrm{H}_{2} \mathrm{O}(10 \mathrm{~mL})$ was added, and the reaction mixture was warmed to rt and extracted with $\mathrm{CH}_{2} \mathrm{Cl}_{2}(3 \times 10 \mathrm{~mL})$. The combined organic phases were dried and concentrated in vacuo. Flash chromatography (3:1 hexanes : EtOAc) yielded unreacted $27(15 \mathrm{mg}, 20 \%)$ and the title compound $59(47 \mathrm{mg}, 63 \%)$ as a red oil: $\mathrm{R}_{\mathrm{f}} 0.28$ (3:1 hexanes : EtOAc); IR: 2953, 2924, 2849, 1700, 1638, 1587, $1561 \mathrm{~cm}^{-1} ;{ }^{1} \mathrm{H}$ NMR (benzene- $\left.d_{6}, 400 \mathrm{MHz}\right): \delta 7.84$ (d, $\left.J=7.6 \mathrm{~Hz}, 1 \mathrm{H}\right), 7.48(\mathrm{~d}, J=7.6 \mathrm{~Hz}$, $1 \mathrm{H}), 6.97(\mathrm{t}, J=7.1 \mathrm{~Hz}, 1 \mathrm{H}), 6.82(\mathrm{t}, J=7.1 \mathrm{~Hz}, 1 \mathrm{H}), 6.73(\mathrm{~d}, J=10.1 \mathrm{~Hz}, 1 \mathrm{H}), 5.57(\mathrm{t}, J=6.3 \mathrm{~Hz}, 1 \mathrm{H}), 4.94(\mathrm{~d}, J=10.1$ $\mathrm{Hz}, 1 \mathrm{H}), 3.94(\mathrm{q}, J=6.6 \mathrm{~Hz}, 2 \mathrm{H}), 2.61(\mathrm{~m}, 2 \mathrm{H}), 1.74(\mathrm{~m}, 3 \mathrm{H}), 1.60(\mathrm{~m}, 1 \mathrm{H}), 1.47(\mathrm{~m}, 1 \mathrm{H}), 1.14(\mathrm{~s}, 3 \mathrm{H}), 0.92(\mathrm{t}, J=6.6 \mathrm{~Hz}$, $1 \mathrm{H}) ;{ }^{13} \mathrm{C}$ NMR (benzene- $\left.d_{6}, 100 \mathrm{MHz}\right) \delta 179.1,175.2,167.2,160.7,142.0,132.8,131.7,131.3,130.9,128.6,128.1,127.9$, 123.7, 123.3, 112.1, 83.4, 60.1, 41.2, 27.2, 24.5, 20.7, 14.2; HRMS (FAB+) Calcd for $\mathrm{C}_{22} \mathrm{H}_{23} \mathrm{O}_{5}(\mathrm{M}+\mathrm{H})^{+}: 367.1545$, Found: 367.1545 . 
Kinetics experiments. Reactions were monitored by ${ }^{1} \mathrm{H}$ NMR at the desired temperature within the NMR probe to $>80 \%$ conversion. Concentration was determined by comparison of alkene protons integration of the starting material and product to an internal standard (1,3,5-trimethoxybenzene). ${ }^{1} \mathrm{H}$ NMR spectra were averages of 2-4 scans separated by $10 \mathrm{~s}$ to ensure accurate integration. Probe temperature was calibrated by measurement of $100 \%$ ethylene glycol spectra.

Electrocyclization of $\mathbf{5 9}$ to $27\left(90^{\circ} \mathrm{C}\right)$

$k\left(2^{\text {nd }}\right.$ order $)=0.000365$

$\mathrm{r}^{2}=0.999625$

$\begin{array}{cccccc}\mathrm{t}(\mathrm{s}) & {[\mathrm{sm}](\mathrm{M})} & 1 /[\mathrm{sm}](1 / \mathrm{M}) & \mathrm{t}(\mathrm{s}) & {[\mathrm{sm}](\mathrm{M})} & 1 /[\mathrm{sm}](1 / \mathrm{M}) \\ 0 & 0.161 & 6.199 & 5580 & 0.0426 & 23.457 \\ 180 & 0.152 & 6.579 & 5760 & 0.0413 & 24.211 \\ 360 & 0.144 & 6.954 & 5940 & 0.0417 & 23.994 \\ 540 & 0.136 & 7.365 & 6120 & 0.0399 & 25.063 \\ 720 & 0.128 & 7.788 & 6300 & 0.0396 & 25.223 \\ 900 & 0.121 & 8.296 & 6480 & 0.0378 & 26.42 \\ 1080 & 0.114 & 8.804 & 6660 & 0.0373 & 26.837 \\ 1260 & 0.107 & 9.331 & 6840 & 0.0371 & 26.984 \\ 1440 & 0.101 & 9.943 & 7020 & 0.0362 & 27.62 \\ 1620 & 0.095 & 10.51 & 7200 & 0.0355 & 28.194 \\ 1800 & 0.09 & 11.09 & 7380 & 0.035 & 28.545 \\ 1980 & 0.086 & 11.66 & 7560 & 0.0336 & 29.73 \\ 2160 & 0.082 & 12.27 & 7740 & 0.0337 & 29.685 \\ 2340 & 0.078 & 12.89 & 7920 & 0.0332 & 30.142 \\ 2520 & 0.074 & 13.43 & 8100 & 0.0327 & 30.58 \\ 2700 & 0.071 & 14.06 & 8280 & 0.0321 & 31.196 \\ 2880 & 0.069 & 14.58 & 8460 & 0.0323 & 30.925 \\ 3060 & 0.066 & 15.23 & 8640 & 0.0306 & 32.635 \\ 3240 & 0.063 & 15.85 & 8820 & 0.0304 & 32.865 \\ 3420 & 0.061 & 16.37 & 9000 & 0.0297 & 33.67 \\ 3600 & 0.058 & 17.18 & 9180 & 0.0298 & 33.52 \\ 3780 & 0.057 & 17.68 & 9360 & 0.0298 & 33.589 \\ 3960 & 0.055 & 18.24 & 9540 & 0.0288 & 34.668 \\ 4140 & 0.053 & 18.87 & 9720 & 0.0286 & 34.973 \\ 4320 & 0.051 & 19.72 & 9900 & 0.0287 & 34.833 \\ 4500 & 0.05 & 20.03 & 10080 & 0.0277 & 36.14 \\ 4680 & 0.049 & 20.57 & 10260 & 0.0275 & 36.345 \\ 4860 & 0.047 & 21.29 & 10440 & 0.0265 & 37.705 \\ 5040 & 0.046 & 21.6 & 10620 & 0.0267 & 37.453 \\ 5220 & 0.045 & 22.4 & 10800 & 0.0264 & 37.87 \\ 5400 & 0.043 & 23.27 & & & \\ & & & & & \end{array}$


Electrocyclization of 59 to $27\left(71^{\circ} \mathrm{C}\right)$

$\begin{array}{cc}\mathrm{t}(\mathrm{s}) & {[\mathrm{sm}]} \\ 0 & 0.07825 \\ 600 & 0.07744 \\ 1200 & 0.07711 \\ 1800 & 0.07653 \\ 2400 & 0.07565 \\ 3000 & 0.07538 \\ 3600 & 0.07485 \\ 4200 & 0.07401 \\ 4800 & 0.07353 \\ 5400 & 0.07301 \\ 6000 & 0.07262 \\ 6600 & 0.07199 \\ 7200 & 0.07155 \\ 7800 & 0.07094 \\ 8400 & 0.07043 \\ 9000 & 0.07021 \\ 9600 & 0.06938 \\ 10200 & 0.06926 \\ 10800 & 0.06871\end{array}$

Electrocyclization of $\mathbf{5 9}$ to 27 with $20 \% \operatorname{EDDA}\left(71^{\circ} \mathrm{C}\right)$

$k_{\text {obs }}\left(1^{\text {st }}\right.$ order $)=0.0004862 \mathrm{~s}^{-1}$

$\mathrm{r}^{2}=0.9987$

$\begin{array}{rrr}\mathrm{t}(\mathrm{s}) & {[\mathbf{5 9}]} & \ln [\mathbf{5 9}] \\ 0 & 0.07555 & -2.583 \\ 300 & 0.065348 & -2.728 \\ 600 & 0.057453 & -2.8568 \\ 900 & 0.049812 & -2.9995 \\ 1200 & 0.043521 & -3.1345 \\ 1500 & 0.038106 & -3.2674 \\ 1800 & 0.033133 & -3.4072 \\ 2100 & 0.028148 & -3.5703 \\ 2400 & 0.0241 & -3.7256 \\ 2700 & 0.020638 & -3.8806 \\ 3000 & 0.017171 & -4.0645 \\ 3300 & 0.01503 & -4.1977 \\ 3600 & 0.012842 & -4.355 \\ 3900 & 0.011025 & -4.5076 \\ 4200 & 0.009814 & -4.624 \\ 4500 & 0.008345 & -4.7861 \\ 4800 & 0.008303 & -4.7912 \\ 4500 & 0.008345 & -4.7861 \\ 4800 & 0.008303 & -4.7912 \\ 5100 & 0.007378 & -4.9092 \\ 5400 & 0.006863 & -4.9816 \\ 5700 & 0.005644 & -5.1771 \\ 6000 & 0.005627 & -5.1802\end{array}$


Anthrakunthone (14)

\begin{tabular}{|l|l|}
\hline lit. ${ }^{1} \mathrm{H}$ NMR values & \\
\hline & found ${ }^{1} \mathrm{H}$ NMR values \\
\hline $8.11(d, J=8.5 \mathrm{~Hz}, 1 \mathrm{H})$ & $9.74(\mathrm{br} \mathrm{s}, 1 \mathrm{H})$ \\
\hline $8.11(d, J=8.0 \mathrm{~Hz}, 1 \mathrm{H})$ & $8.10(\mathrm{~d}, J=4.2 \mathrm{~Hz}, 1 \mathrm{H})$ \\
\hline $7.58(d, J=2.5 \mathrm{~Hz}, 1 \mathrm{H})$ & $8.08(\mathrm{~d}, J=3.4 \mathrm{~Hz}, 1 \mathrm{H})$ \\
\hline $7.52(d, J=8.0 \mathrm{~Hz}, 1 \mathrm{H})$ & $7.68(\mathrm{~d}, J=8.1 \mathrm{~Hz}, 1 \mathrm{H})$ \\
\hline $7.16(d d, J=2.5,8.5 \mathrm{~Hz}, 1 \mathrm{H})$ & $7.59(\mathrm{~m}, 1 \mathrm{H})$ \\
\hline $3.06(t, J=7.5 \mathrm{~Hz}, 2 \mathrm{H})$ & $7.26(\mathrm{~m}, 1 \mathrm{H})$ \\
\hline $2.77(t, J=7.5 \mathrm{~Hz}, 2 \mathrm{H})$ & $3.03(\mathrm{~m}, 2 \mathrm{H})$ \\
\hline $2.72(s, 3 \mathrm{H})$ & $2.82-2.89(\mathrm{~m}, 2 \mathrm{H})$ \\
\hline $2.20(s, 3 \mathrm{H})$ & $2.75(\mathrm{~s}, 3 \mathrm{H})$ \\
\hline
\end{tabular}

Isopinnatal (9)

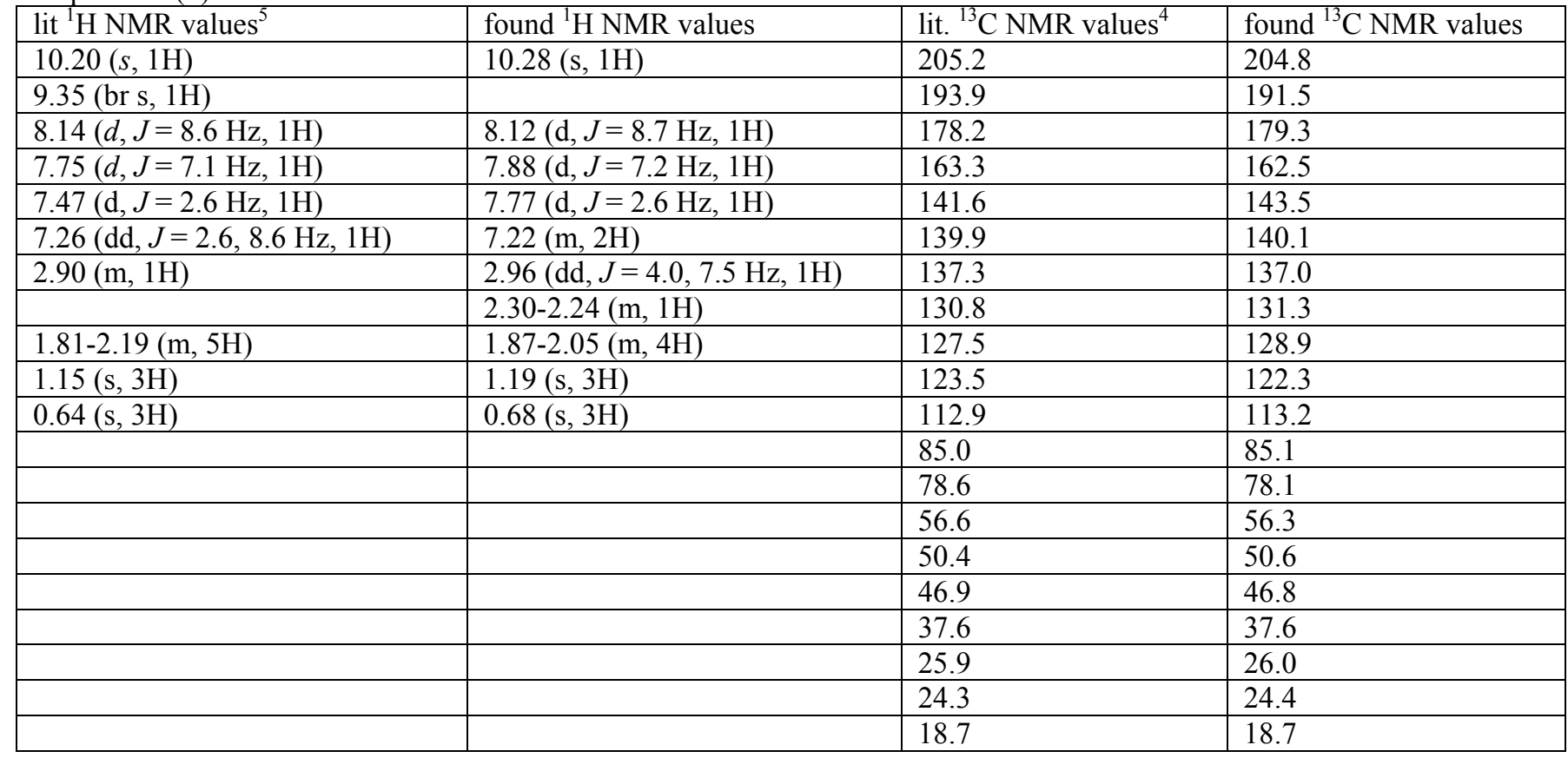

Sterekunthal B (10)

\begin{tabular}{|l|l|}
\hline lit. ${ }^{1} \mathrm{H}$ NMR values & \\
\hline $10.27(\mathrm{~s}, 1 \mathrm{H})$ & found ${ }^{1} \mathrm{H}$ NMR values \\
\hline $8.28(\mathrm{dd}$, & $10.25(\mathrm{~s}, 1 \mathrm{H})$ \\
\hline 8.15 & $8.26(\mathrm{~d}, J=7.6 \mathrm{~Hz}, 1 \mathrm{H})$ \\
\hline 7.88 & $8.13(\mathrm{~d}, J=7.6 \mathrm{~Hz}, 1 \mathrm{H})$ \\
\hline 7.82 & $7.87(\mathrm{~d}, J=7.1 \mathrm{~Hz}, 1 \mathrm{H})$ \\
\hline 7.76 & $7.78(\mathrm{~m}, 2 \mathrm{H})$ \\
\hline 2.96 & \\
\hline 2.28 & $2.96(\mathrm{dd}, J=3.8,7.1 \mathrm{~Hz}, 1 \mathrm{H})$ \\
\hline $1.85-2.05$ & $2.25(\mathrm{~m}, 1 \mathrm{H})$ \\
\hline 1.77 & $1.82-2.07(\mathrm{~m}, 4 \mathrm{H})$ \\
\hline 1.19 & \\
\hline 0.68 & $1.17(\mathrm{~s}, 3 \mathrm{H})$ \\
\hline & $0.66(\mathrm{~s}, 3 \mathrm{H})$ \\
\hline
\end{tabular}


Pyranokunthone A (15)

\begin{tabular}{|c|c|c|c|}
\hline lit. ${ }^{1}$ H NMR values ${ }^{4}$ & found ${ }^{1} \mathrm{H}$ NMR values & lit. ${ }^{13} \mathrm{C}$ NMR values ${ }^{4}$ & found ${ }^{13} \mathrm{C}$ NMR values \\
\hline $7.89(\mathrm{~d}, J=8.0 \mathrm{~Hz}, 1 \mathrm{H})$ & $7.99(\mathrm{~d}, J=8.4 \mathrm{~Hz}, 1 \mathrm{H})$ & 185 & 184.8 \\
\hline $7.40(\mathrm{~d}, J=2.5 \mathrm{~Hz}, 1 \mathrm{H})$ & $7.44(\mathrm{~d}, J=2.7 \mathrm{~Hz}, 1 \mathrm{H})$ & 181 & 183.0 \\
\hline $7.04(\mathrm{dd}, J=2.5,8.0 \mathrm{~Hz}, 1 \mathrm{H})$ & $7.04(\mathrm{dd}, J=2.7,8.4 \mathrm{~Hz}, 1 \mathrm{H})$ & 161 & 161.9 \\
\hline $6.03(\mathrm{~d}, J=2.0 \mathrm{~Hz}, 1 \mathrm{H})$ & $5.90(\mathrm{~s}, 1 \mathrm{H})$ & 137 & 135.8 \\
\hline 3.45 (br s, $1 \mathrm{H})$ & 3.51 (br s, $1 \mathrm{H})$ & 134 & 135.0 \\
\hline $1.70(\mathrm{~m}, 1 \mathrm{H})$ & $1.72-1.80(\mathrm{~m}, 1 \mathrm{H})$ & 124 & 124.1 \\
\hline $1.60(\mathrm{~s}, 3 \mathrm{H})$ & $1.67(\mathrm{~s}, 3 \mathrm{H})$ & 122 & 123.2 \\
\hline $1.48(\mathrm{~s}, 3 \mathrm{H})$ & $1.33(\mathrm{~s}, 3 \mathrm{H})$ & 121 & 120.4 \\
\hline $1.25(\mathrm{~s}, 3 \mathrm{H})$ & $1.25(\mathrm{~m}, 5 \mathrm{H})$ & 113 & 112.6 \\
\hline \multirow[t]{4}{*}{$1.22(\mathrm{~m}, 2 \mathrm{H})$} & & 81 & 80.2 \\
\hline & & 25 & 24.8 \\
\hline & & 24 & 23.6 \\
\hline & & 21 & 20.4 \\
\hline
\end{tabular}

Pyranokunthone B (16)

\begin{tabular}{|l|l|}
\hline lit. $^{1} \mathrm{H}$ NMR values & \\
\hline $8.01(\mathrm{~d}, J=8.5 \mathrm{~Hz}, 1 \mathrm{H})$ & found $^{\mathrm{l}} \mathrm{H}$ NMR values \\
\hline & $8.00(\mathrm{~d}, J=8.4 \mathrm{~Hz}, 1 \mathrm{H})$ \\
\hline $7.52(\mathrm{~d}, J=2.5 \mathrm{~Hz}, 1 \mathrm{H})$ & $7.81(\mathrm{br} \mathrm{s}, 1 \mathrm{H})$ \\
\hline $7.14(\mathrm{dd}, J=2.5,8.5 \mathrm{~Hz}, 1 \mathrm{H})$ & $7.63(\mathrm{~d}, \mathrm{~J}=2.6 \mathrm{~Hz}, 1 \mathrm{H})$ \\
\hline $6.68(\mathrm{~d}, J=10.0 \mathrm{~Hz}, 1 \mathrm{H})$ & $6.20(\mathrm{dd}, \mathrm{J}=2.6,8.4 \mathrm{~Hz}, 1 \mathrm{H})$ \\
\hline $5.67(\mathrm{~d}, J=10.0 \mathrm{~Hz}, 1 \mathrm{H})$ & $5.67(\mathrm{~d}, \mathrm{~J}=10.1 \mathrm{~Hz}, 1 \mathrm{H})$ \\
\hline $5.07(\mathrm{t}, J=7.0 \mathrm{~Hz}, 1 \mathrm{H})$ & $5.03(\mathrm{~m}, 1 \mathrm{H})$ \\
\hline $2.10(\mathrm{~m}, 1 \mathrm{H})$ & $2.07(\mathrm{~m}, 2 \mathrm{H})$ \\
\hline $1.92(\mathrm{~m}, 2 \mathrm{H})$ & $1.90(\mathrm{~m}, 1 \mathrm{H})$ \\
\hline $1.71(\mathrm{~m}, 1 \mathrm{H})$ & $1.65(\mathrm{~m}, 1 \mathrm{H})$ \\
\hline $1.63(\mathrm{~s}, 3 \mathrm{H})$ & $1.60(\mathrm{~s}, 3 \mathrm{H})$ \\
\hline $1.55(\mathrm{~s}, 3 \mathrm{H})$ & $1.52(\mathrm{~s}, 3 \mathrm{H})$ \\
\hline $1.50(\mathrm{~s}, 3 \mathrm{H})$ & $1.48(\mathrm{~s}, 3 \mathrm{H})$ \\
\hline
\end{tabular}

\section{Notes and References}

${ }^{1}$ Kasturi, T. R.; Arunacha.T Can. J. Chem. 1966, 44, 1086-1089.

${ }^{2}$ De Min, M.; Croux, S.; Tournaire, C.; Hocquaux, M.; Jacquet, B.; Oliveros, E.; Maurette, M. T. Tetrahedron 1992, 48, 1869-1882.

${ }^{3}$ We believe one signal is obscured by residual solvent.

${ }^{4}$ Onegi, B.; Kraft, C.; Kohler, I.; Freund, M.; Jenett-Siems, K.; Siems, K.; Beyer, G.; Melzig, M. F.; Bienzle, U.; Eich, E. Phytochemistry 2002, 60, 39-44.

${ }^{5}$ Akunyili, D. N.; Houghton, P. J. Phytochemistry 1993, 32, 1015-1018. 


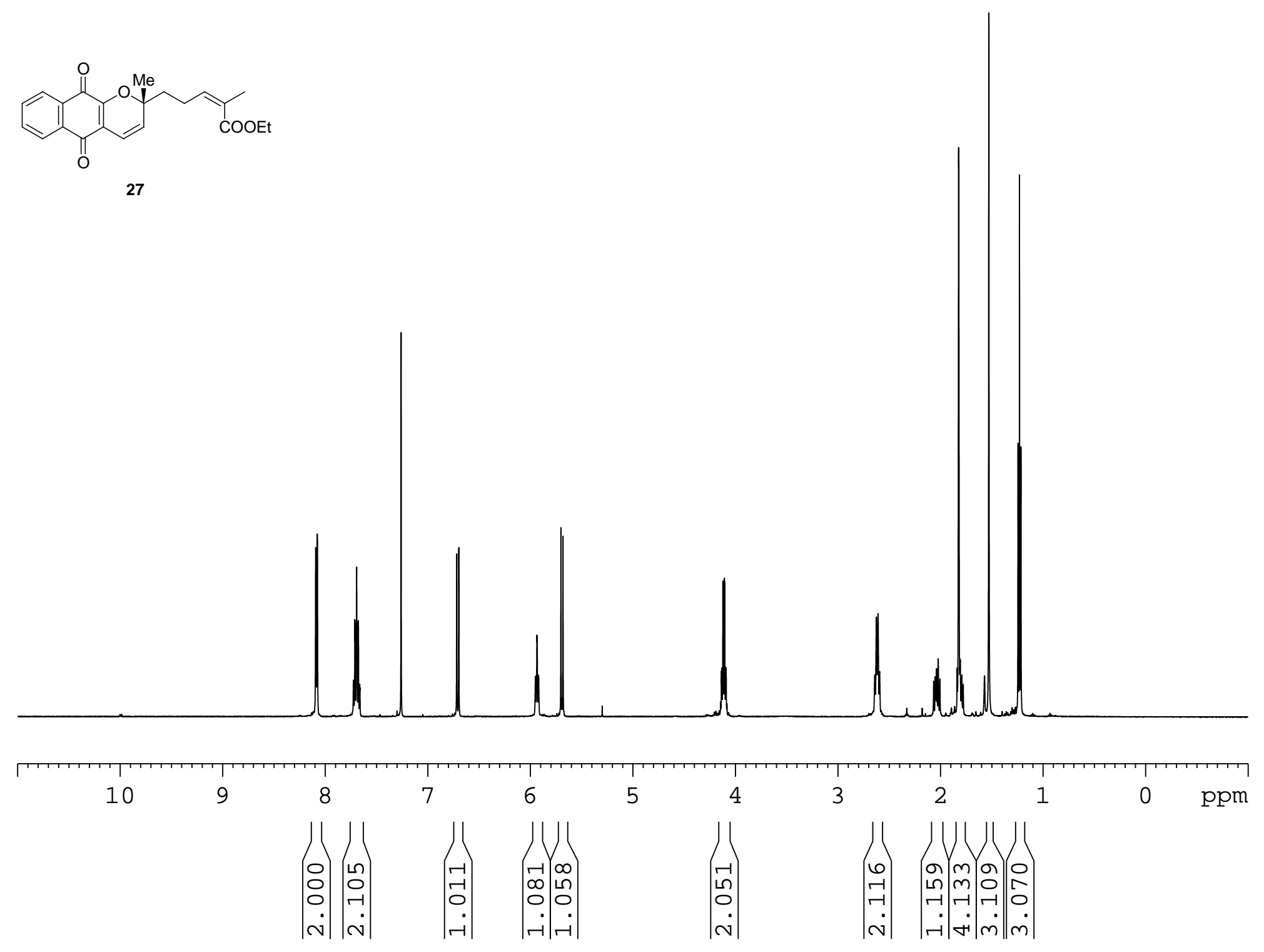



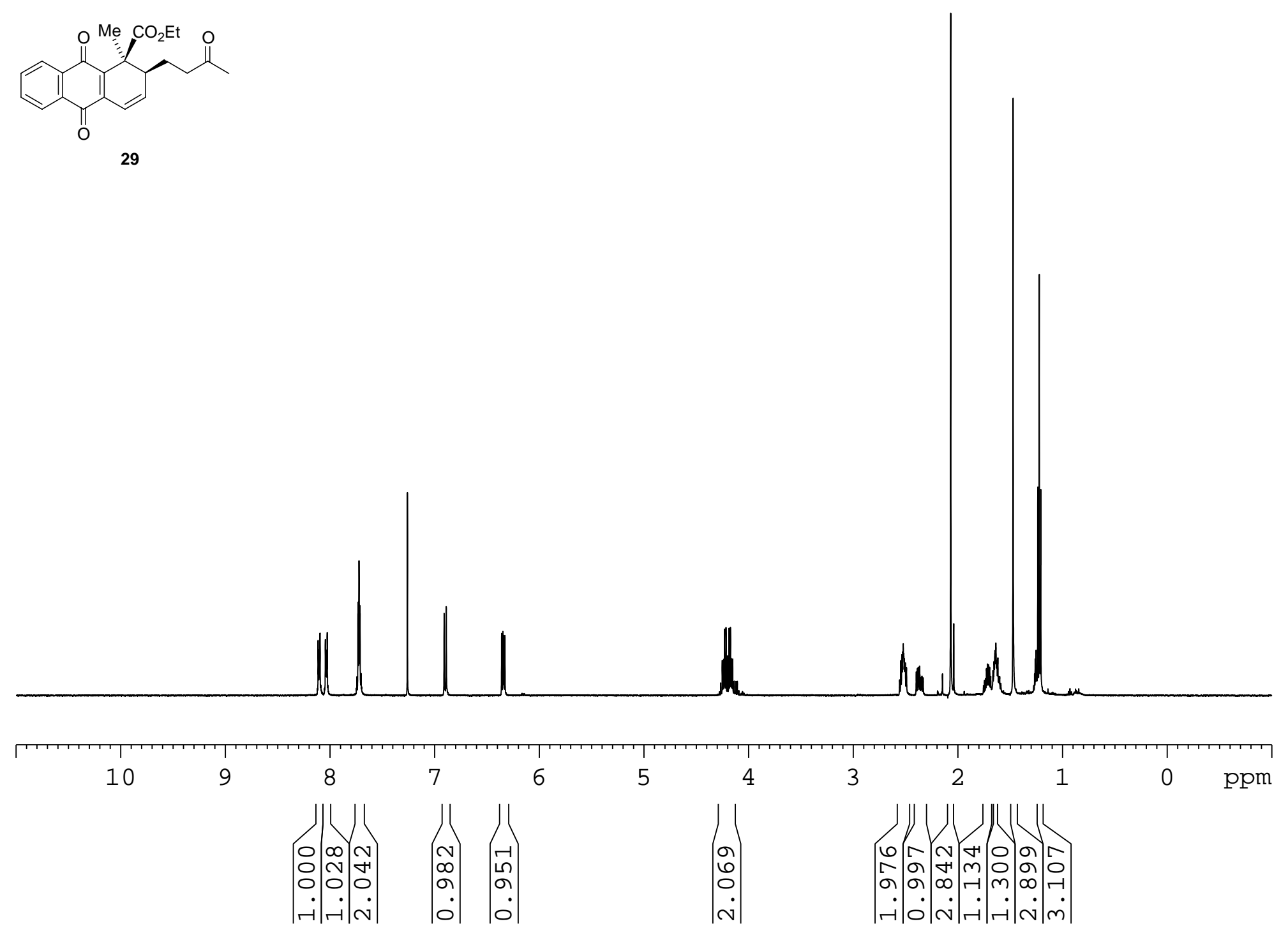

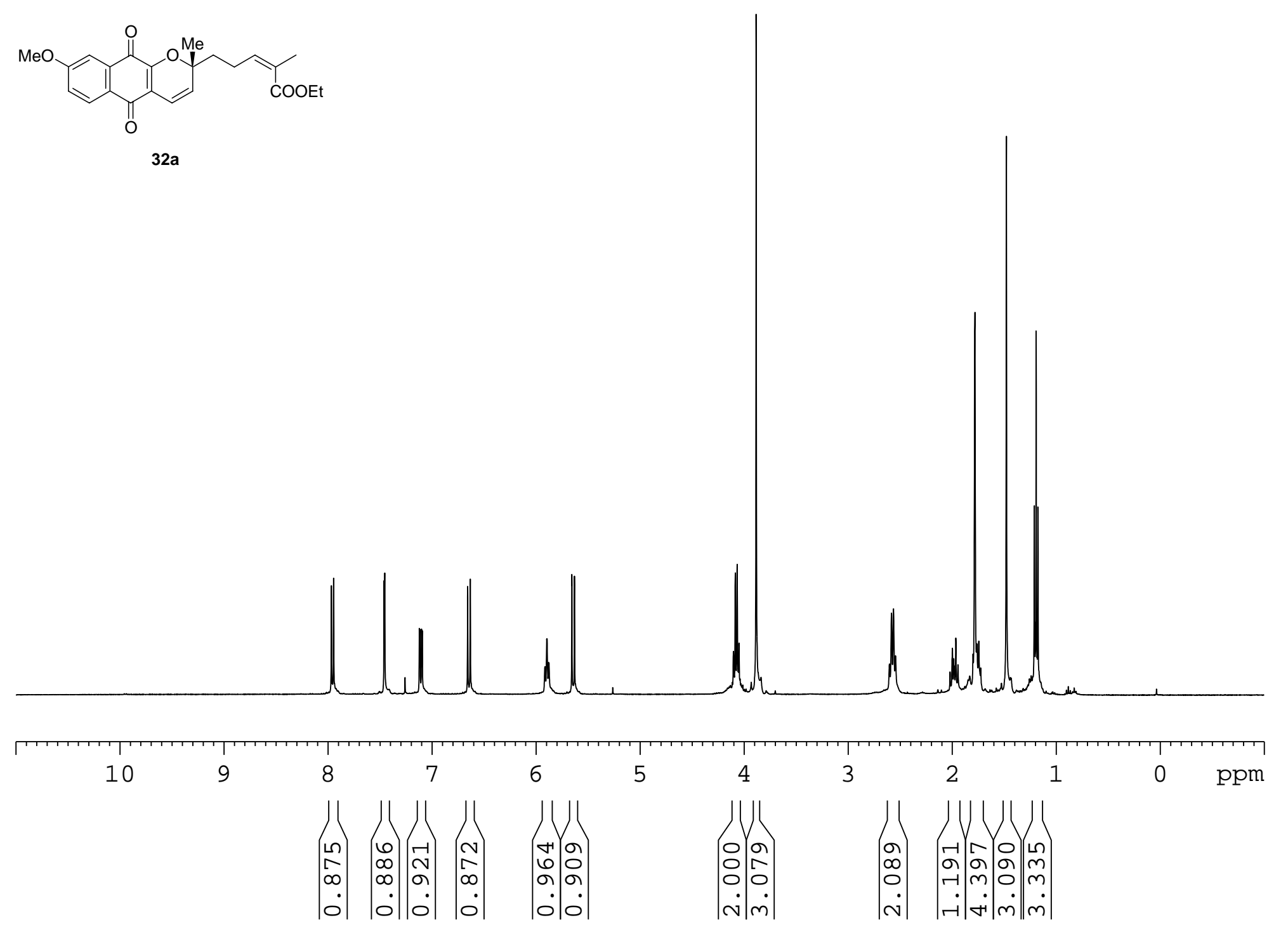

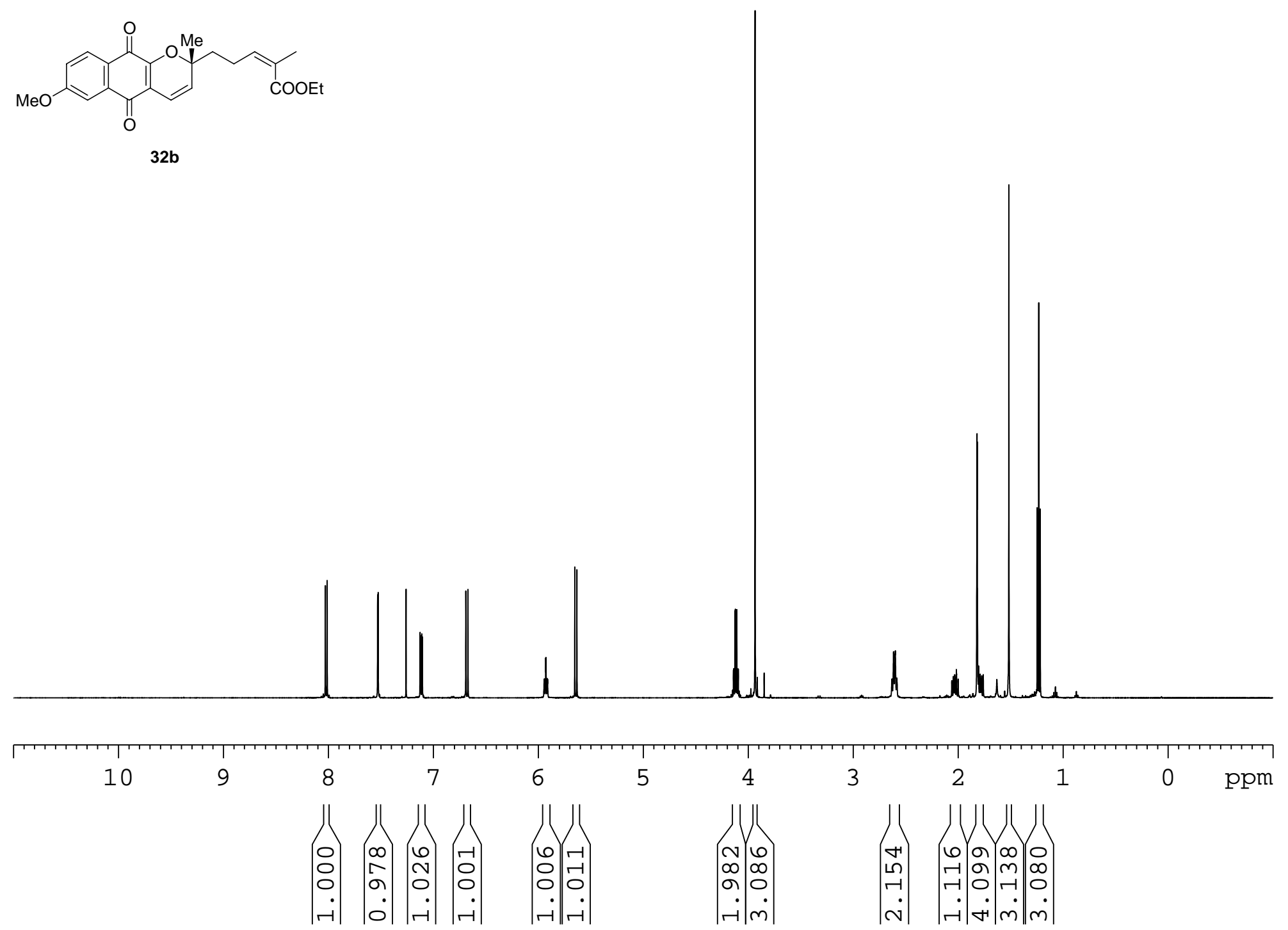

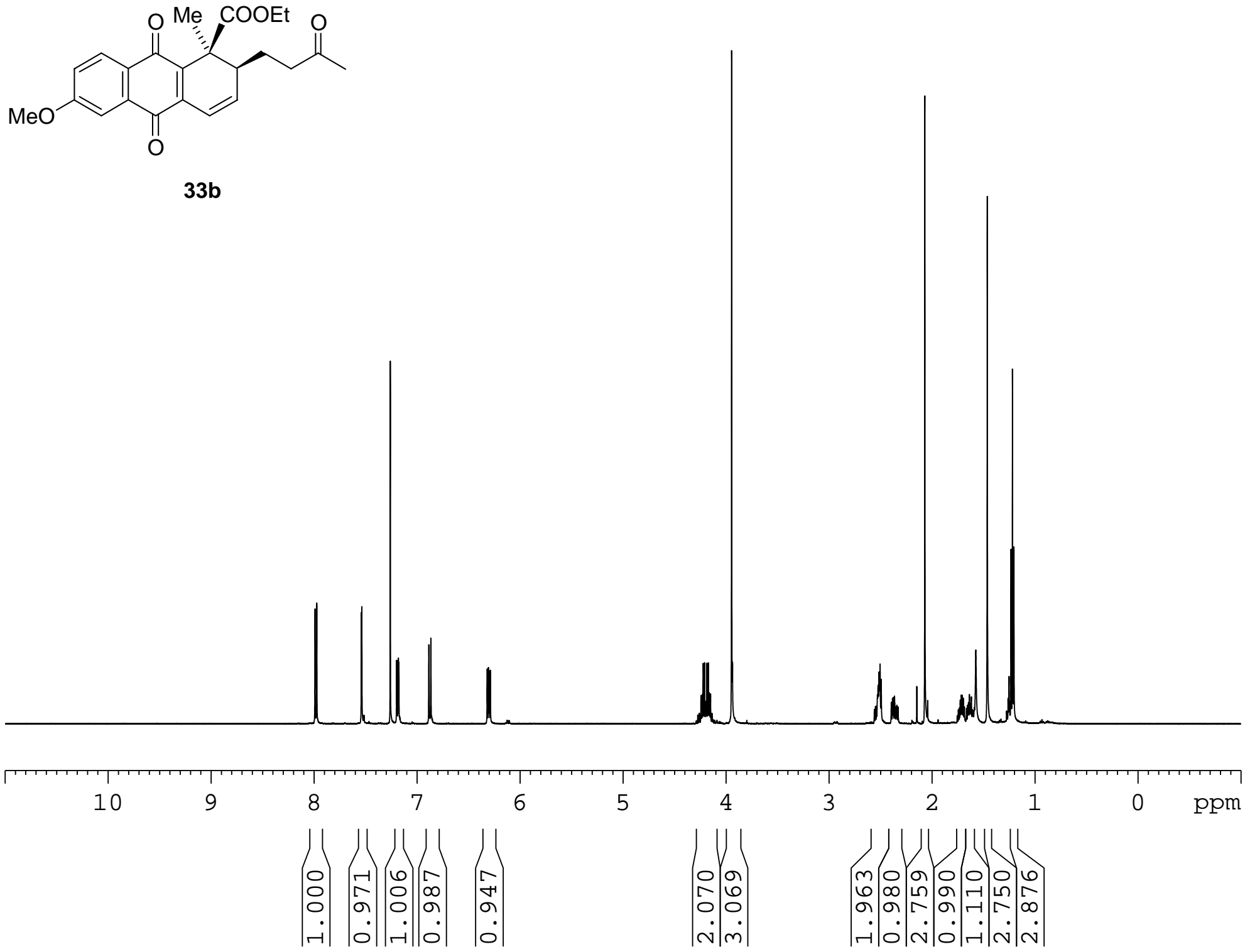

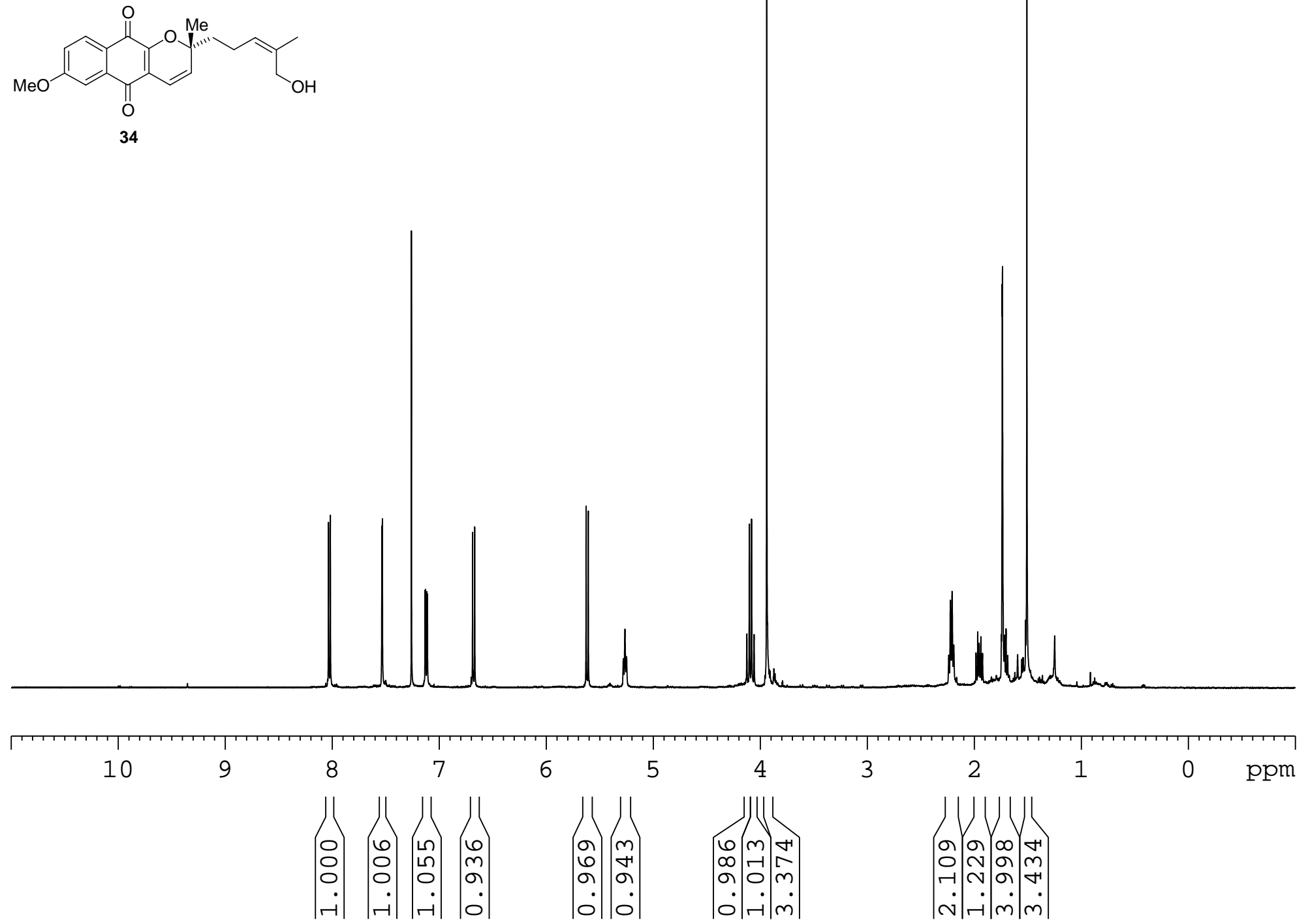

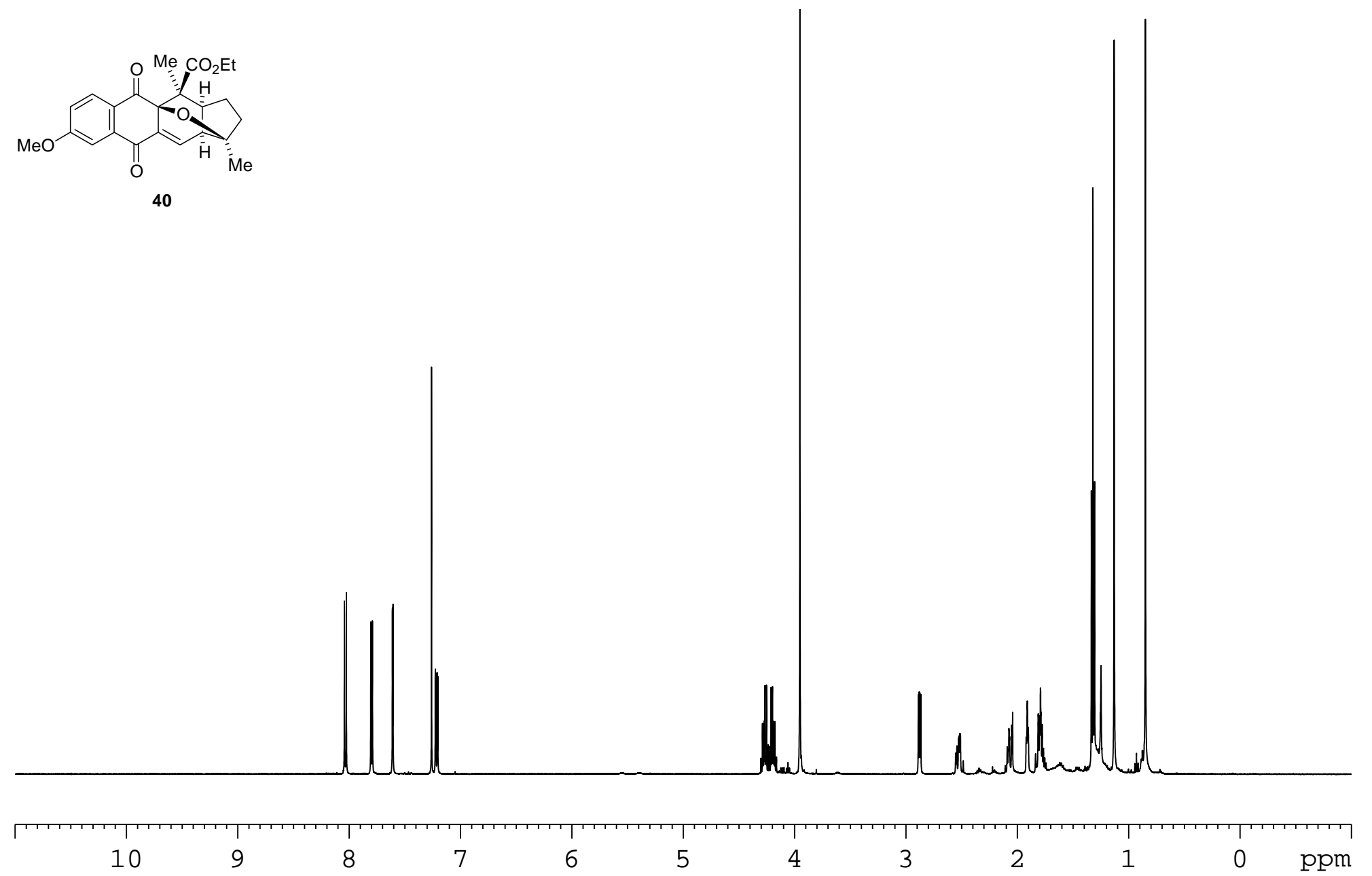


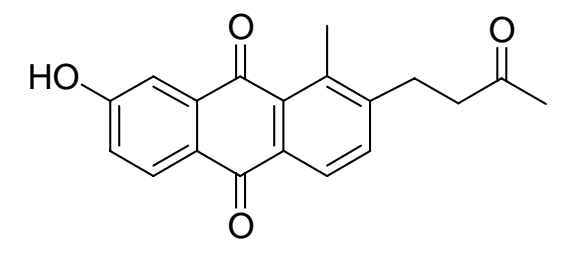

anthrakunthone (14)

(acetone- $d_{6}$ )
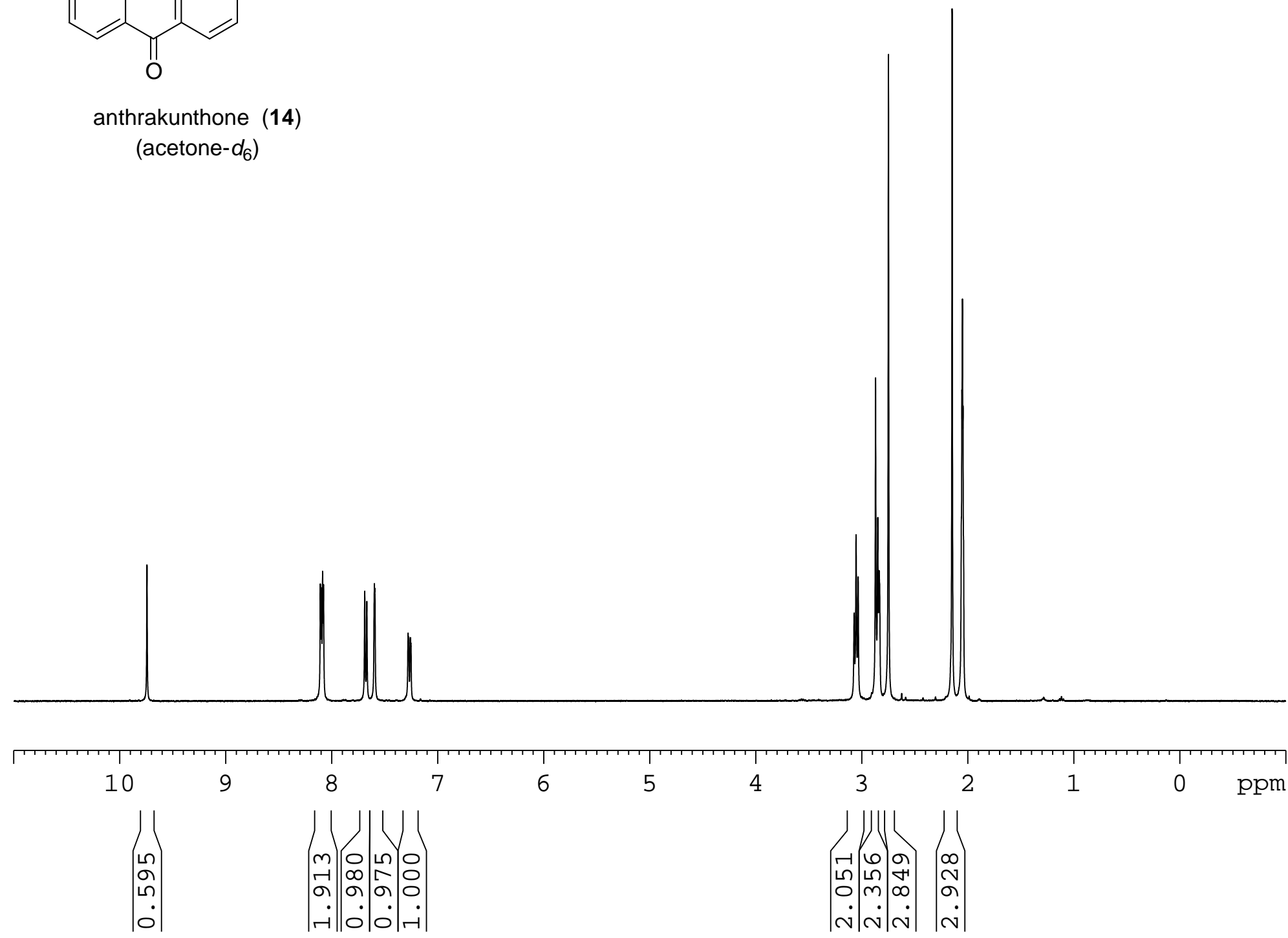

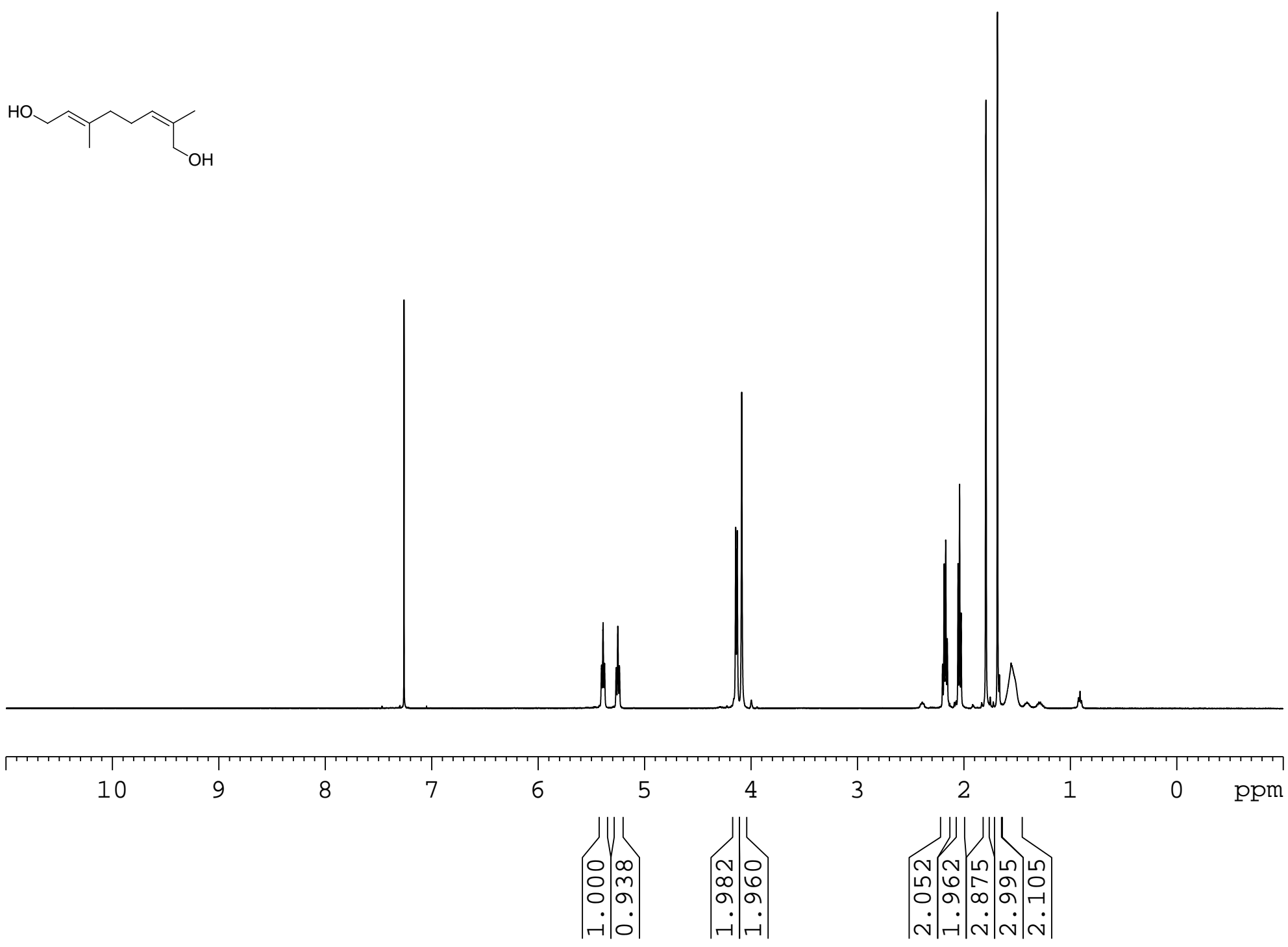

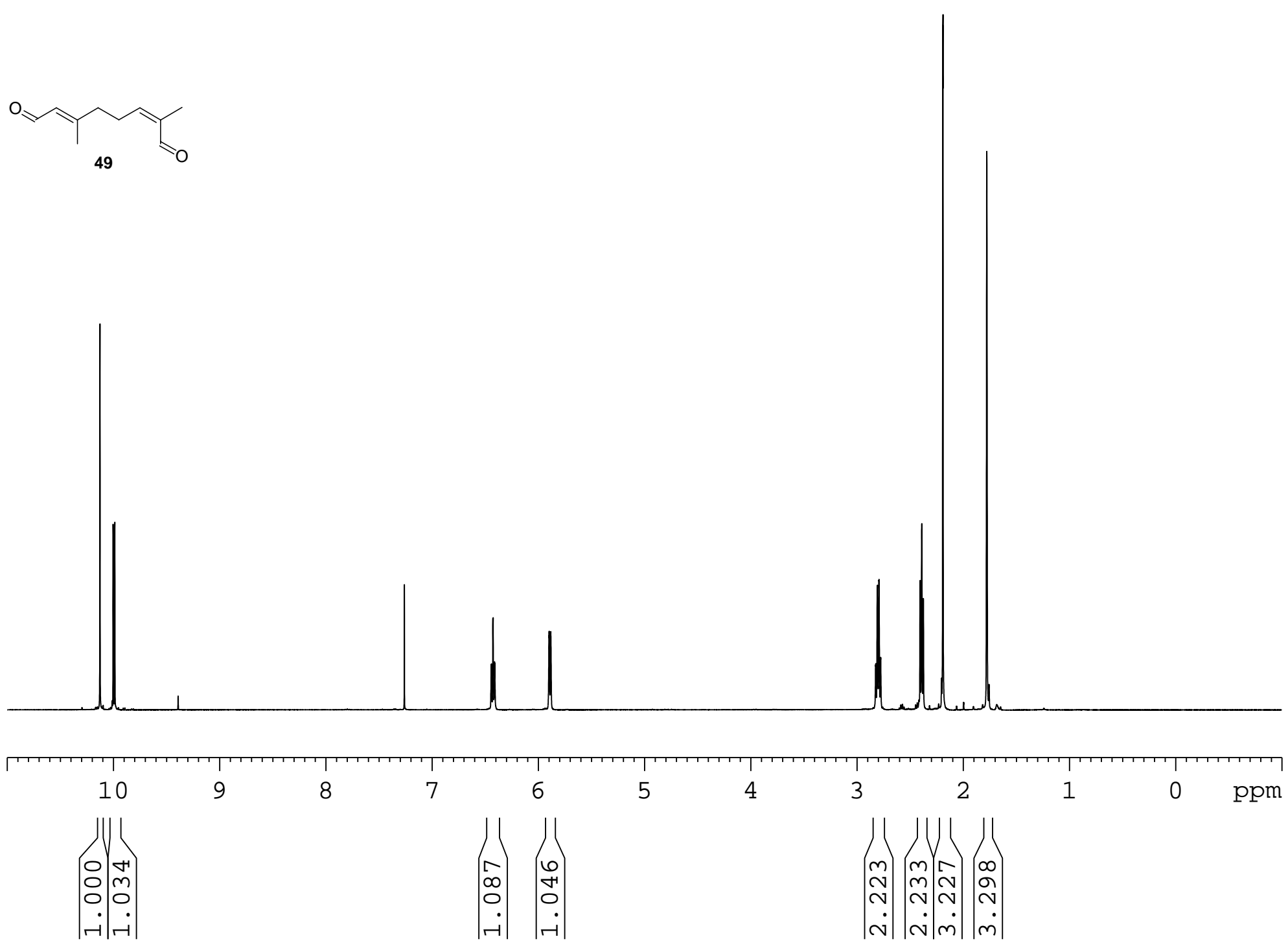

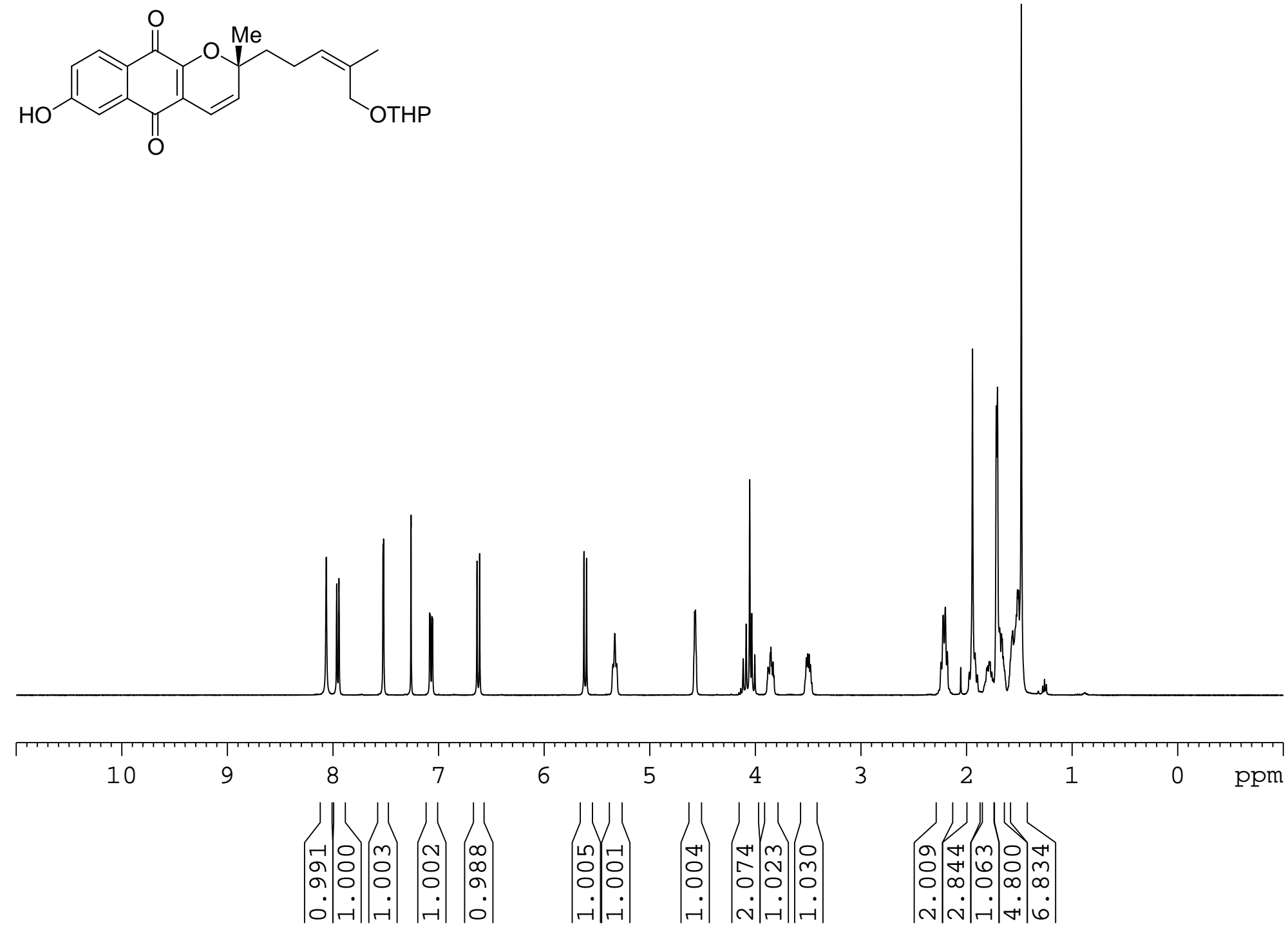

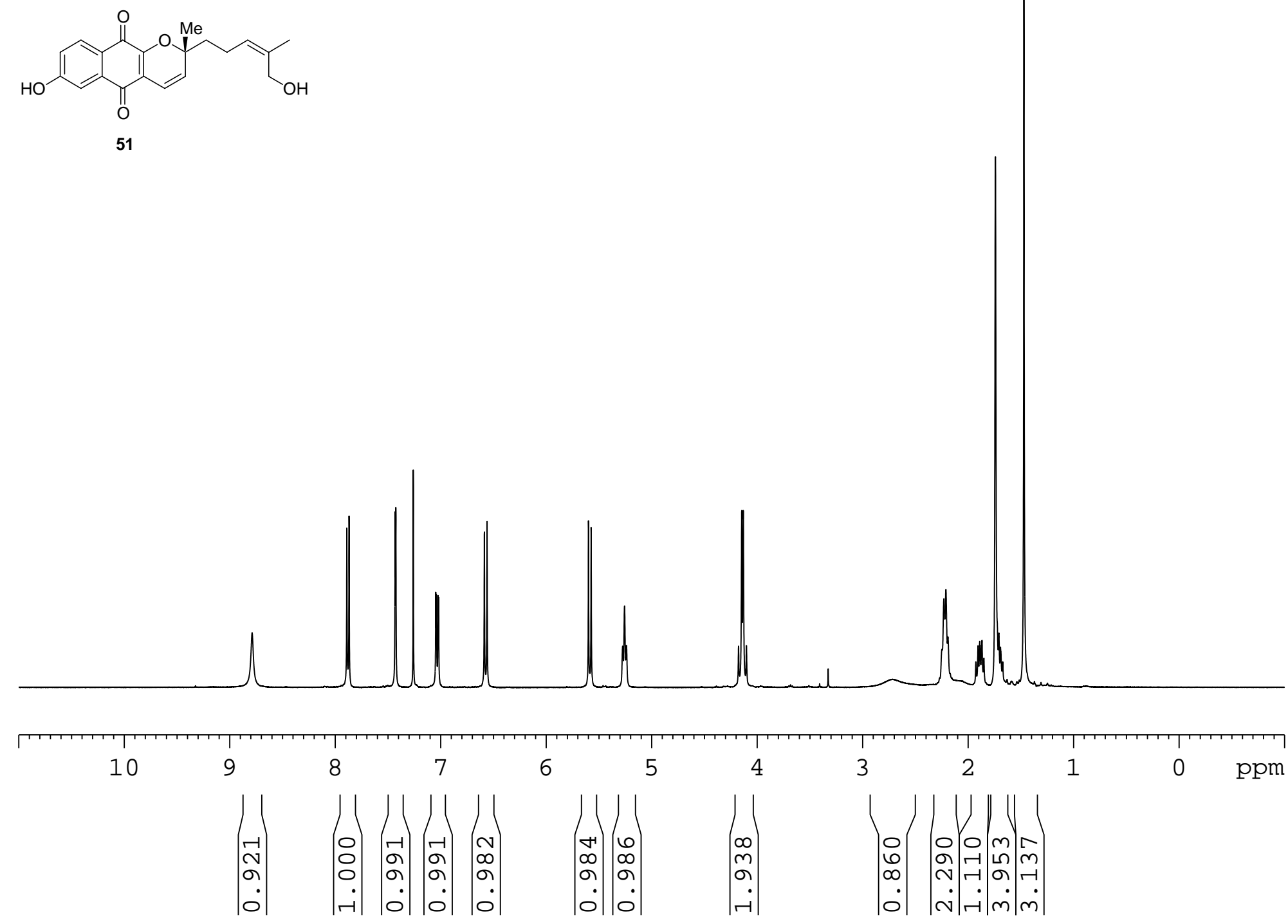

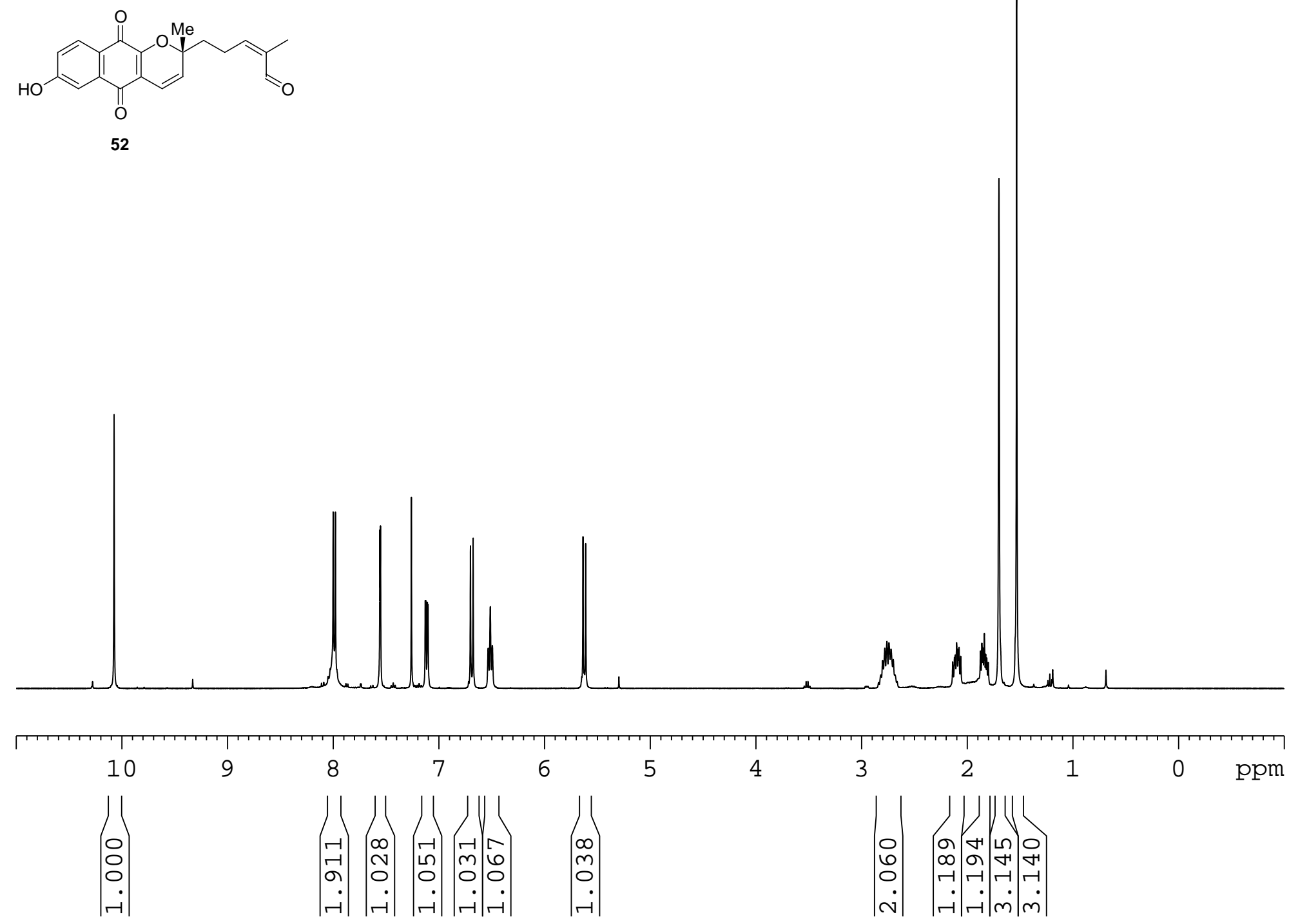

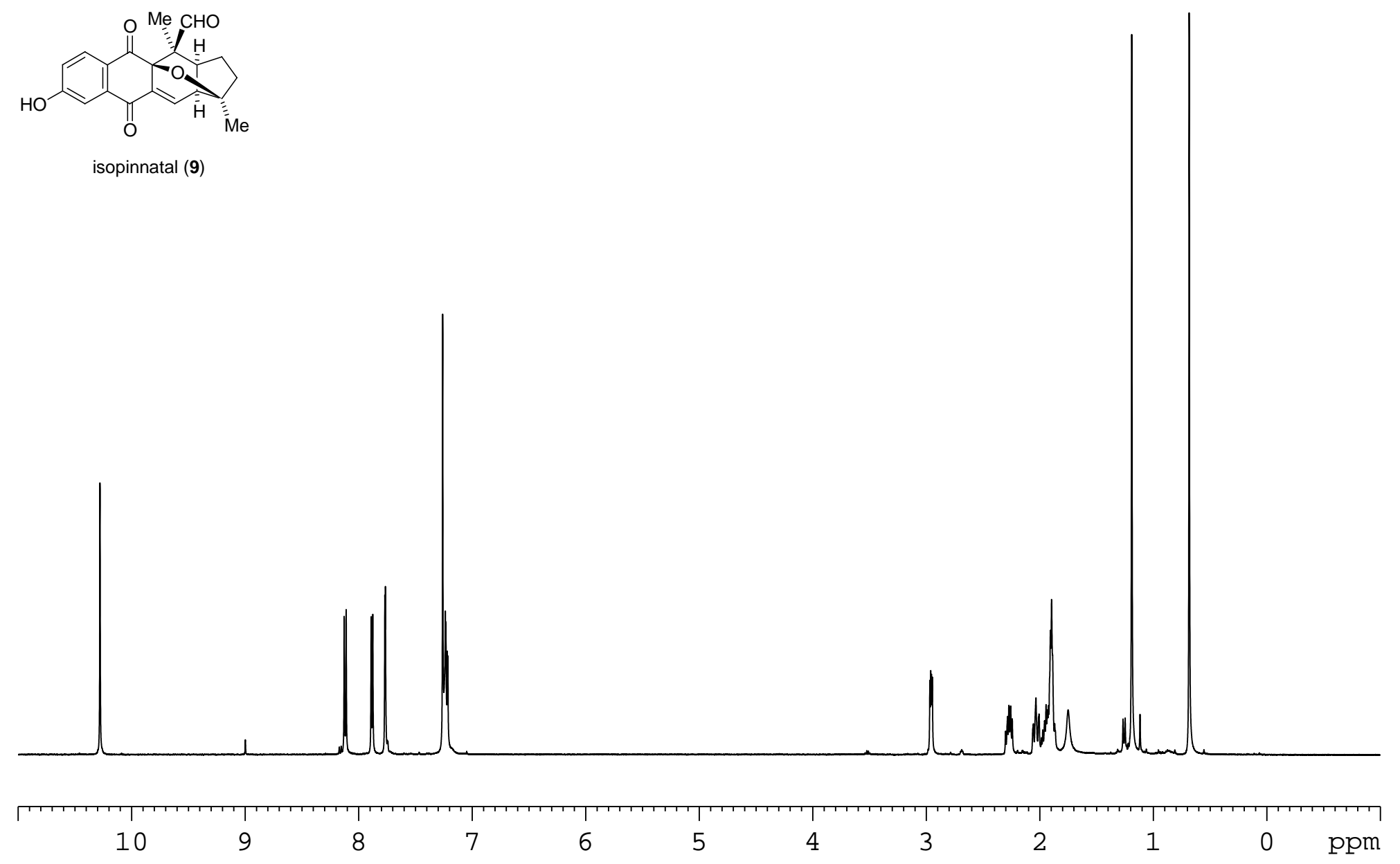

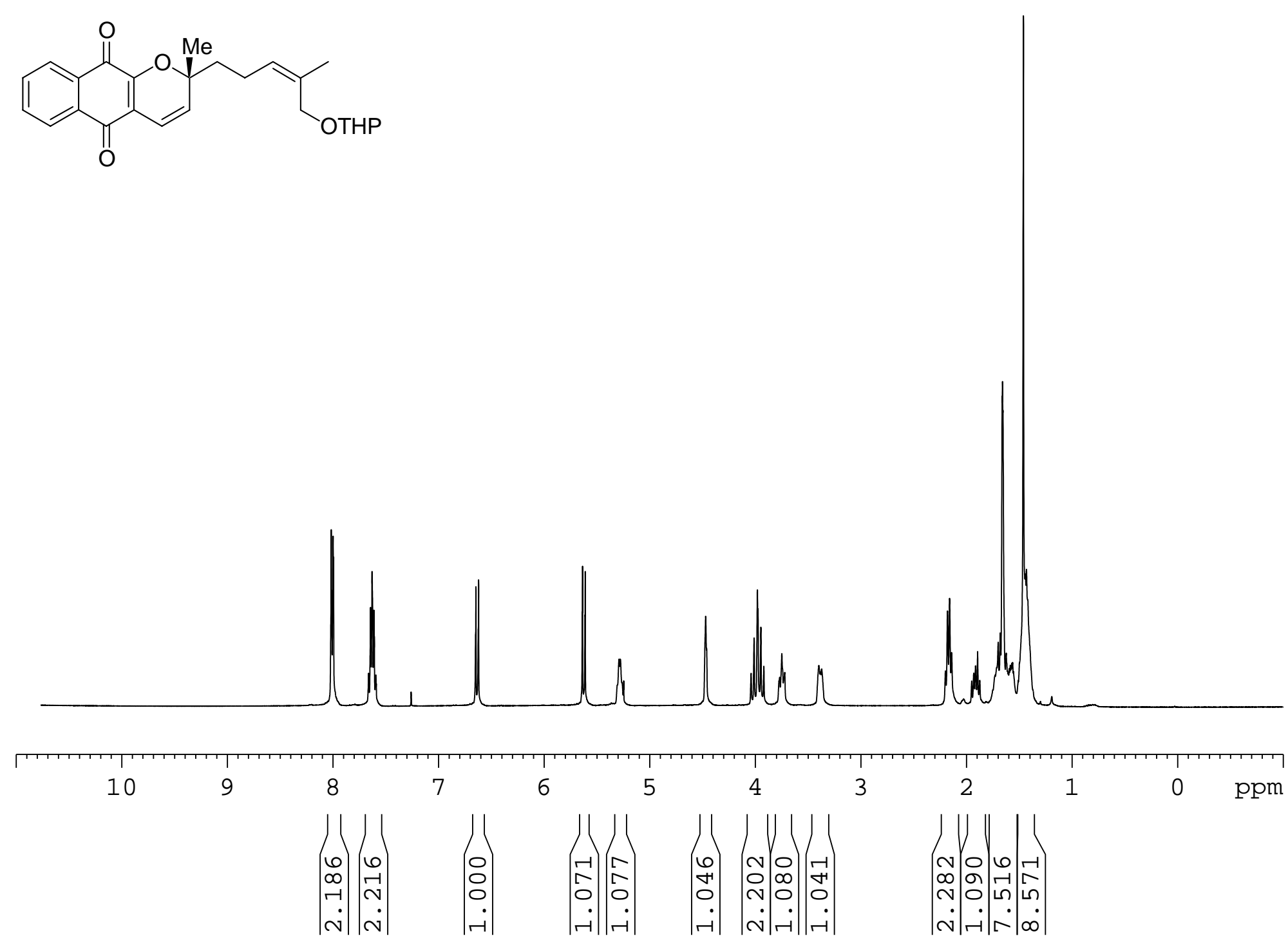

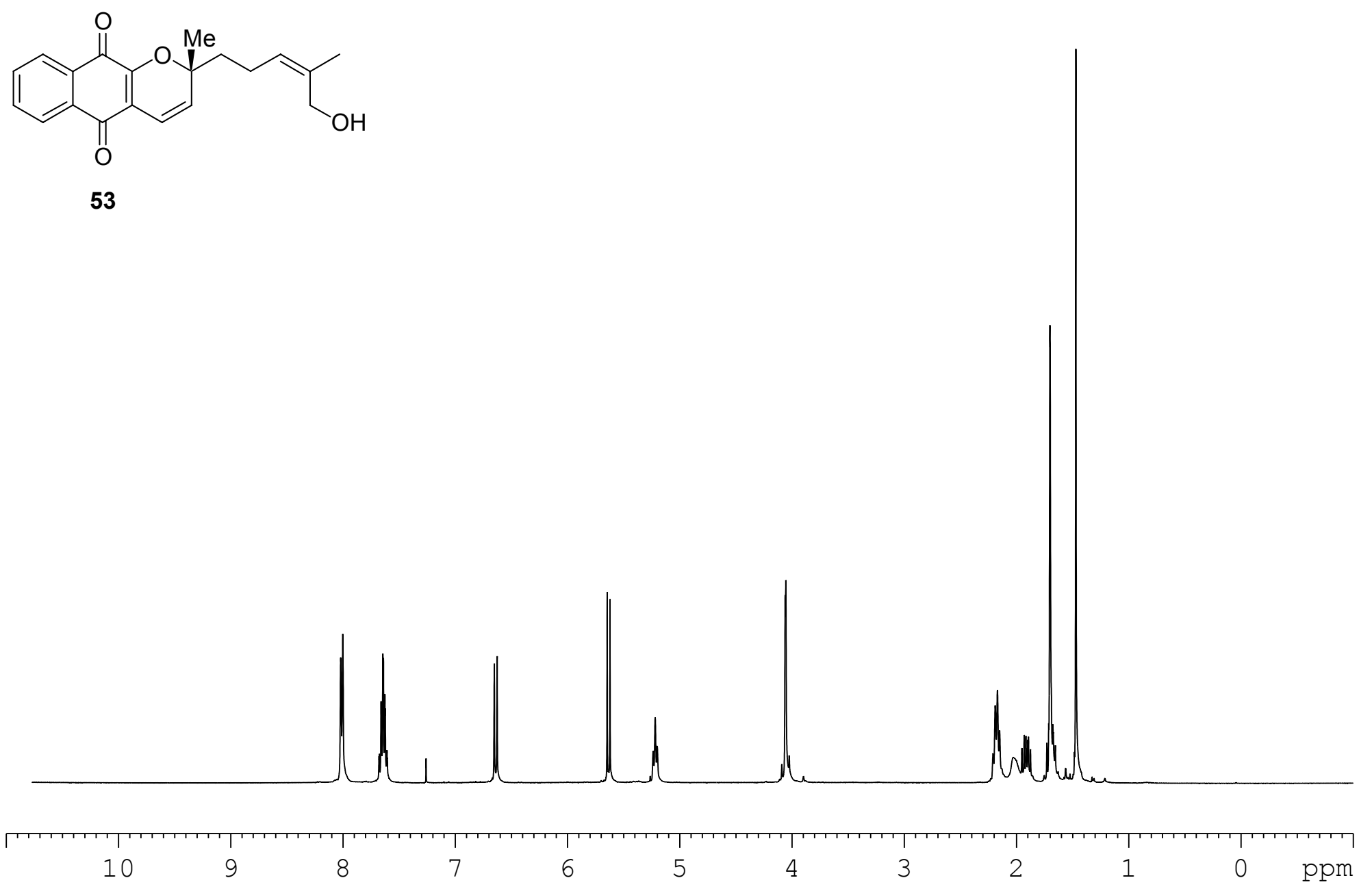

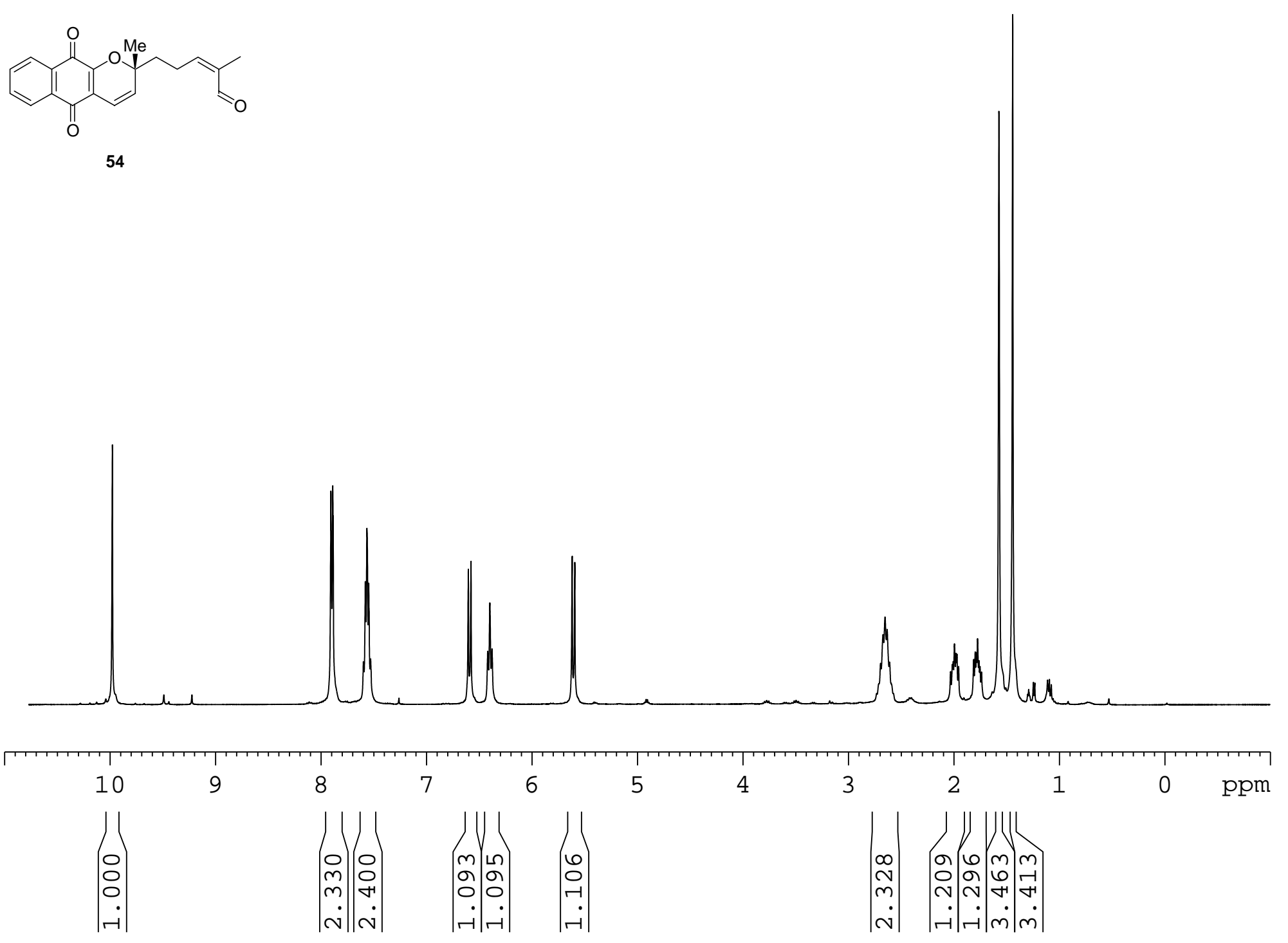


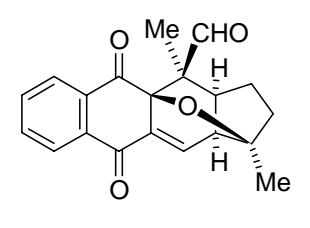

sterekunthal B (10)

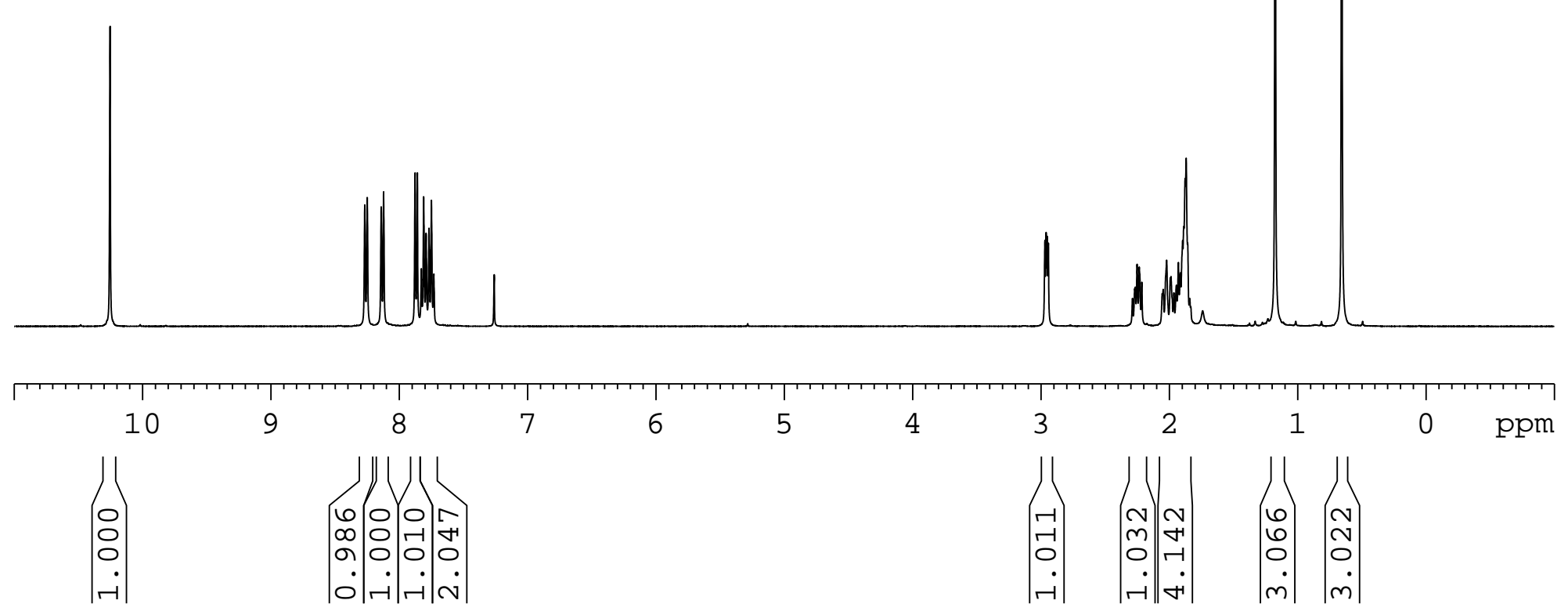




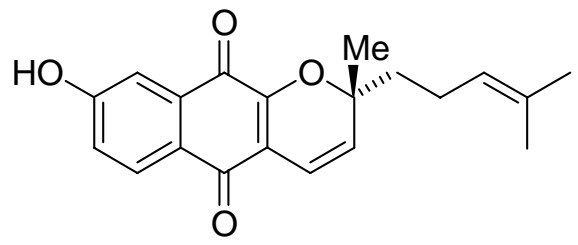

pyranokunthone B (16)

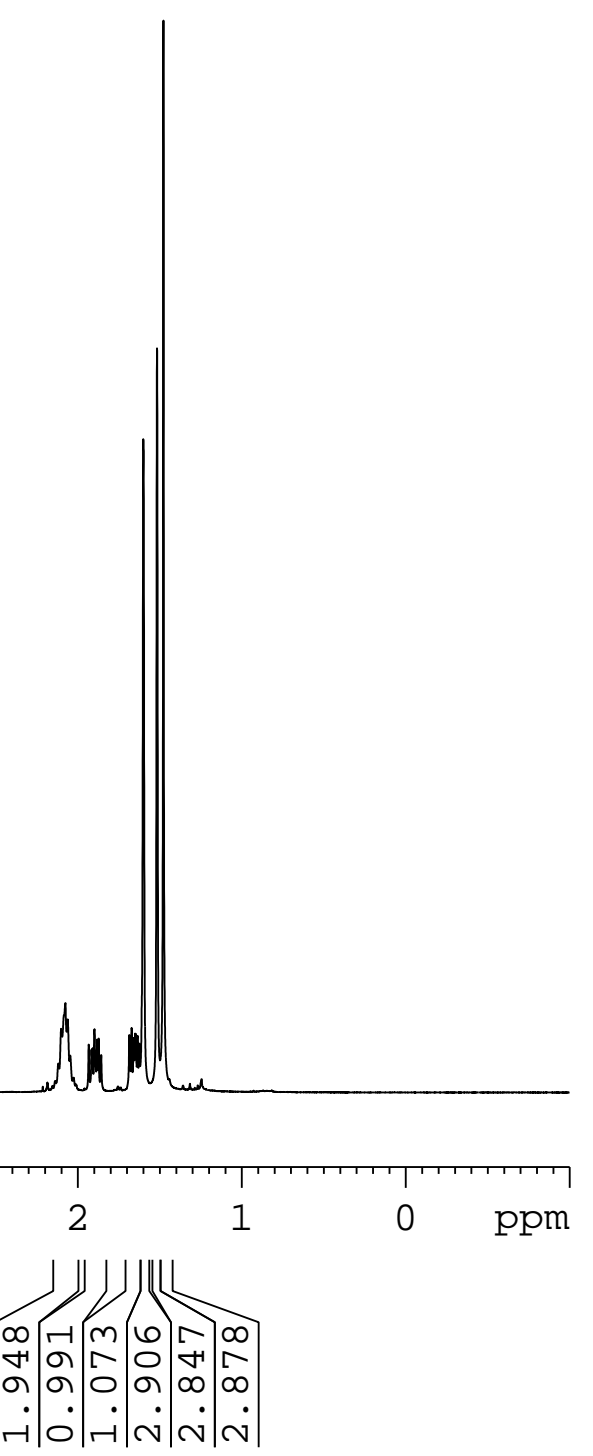




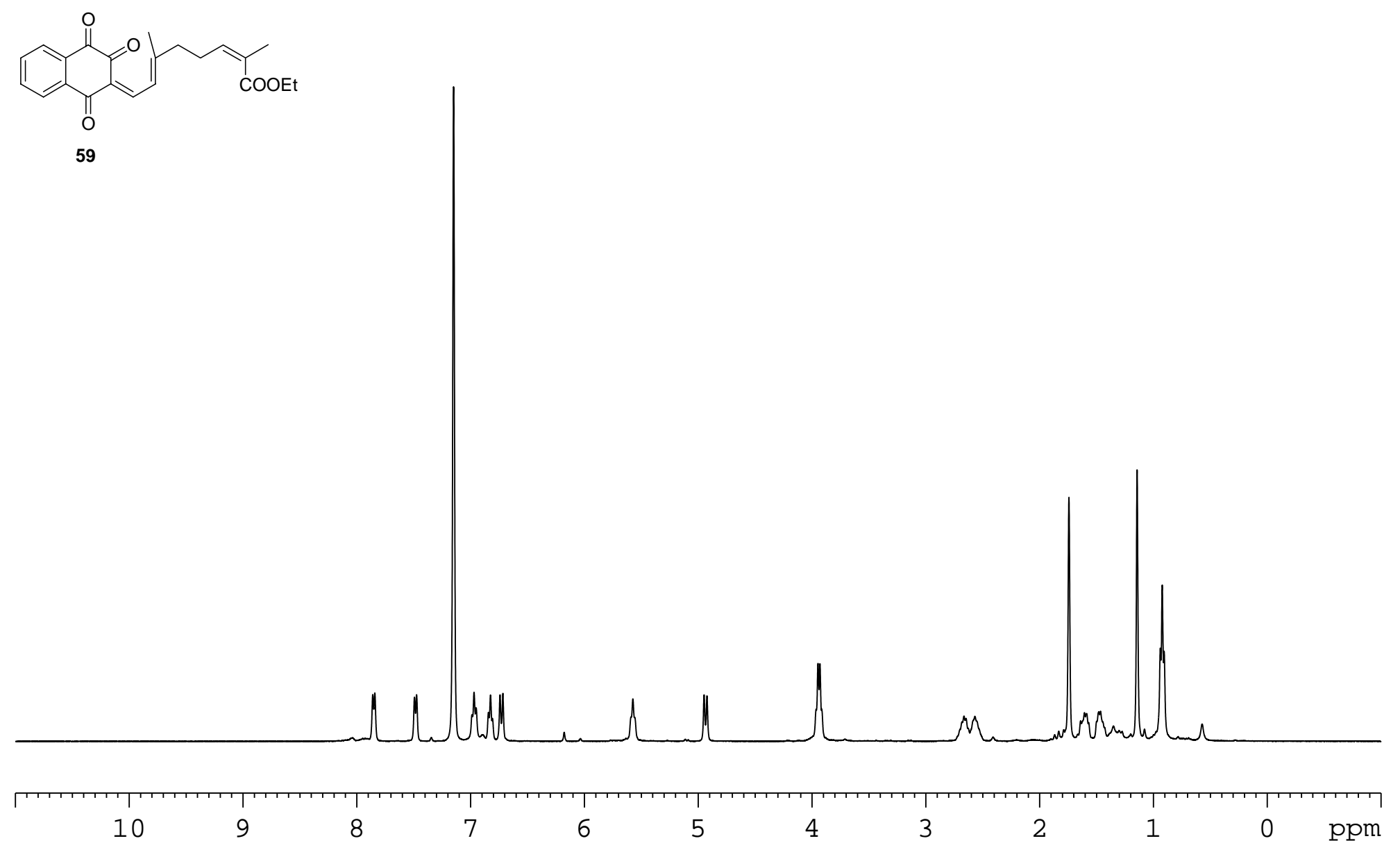



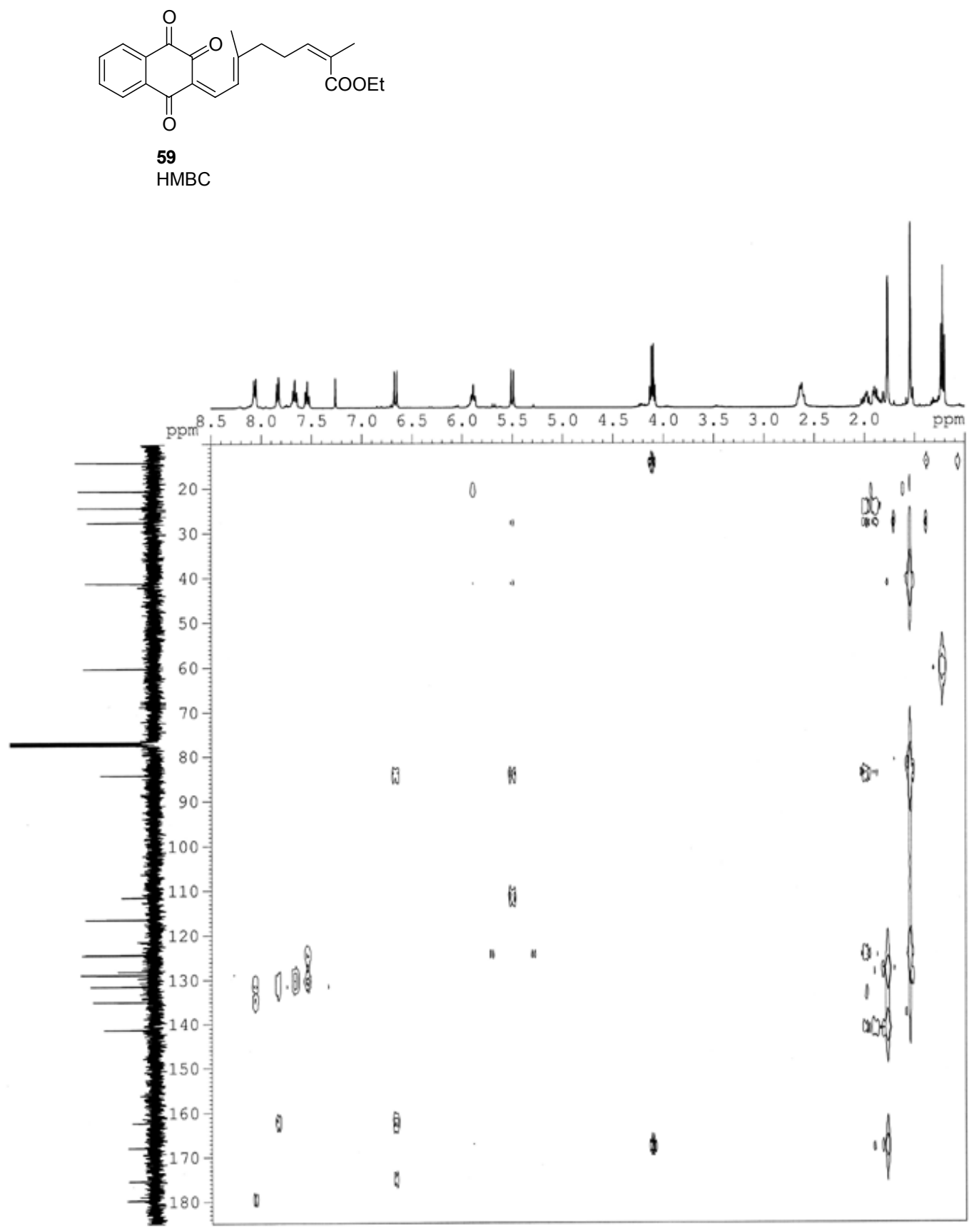


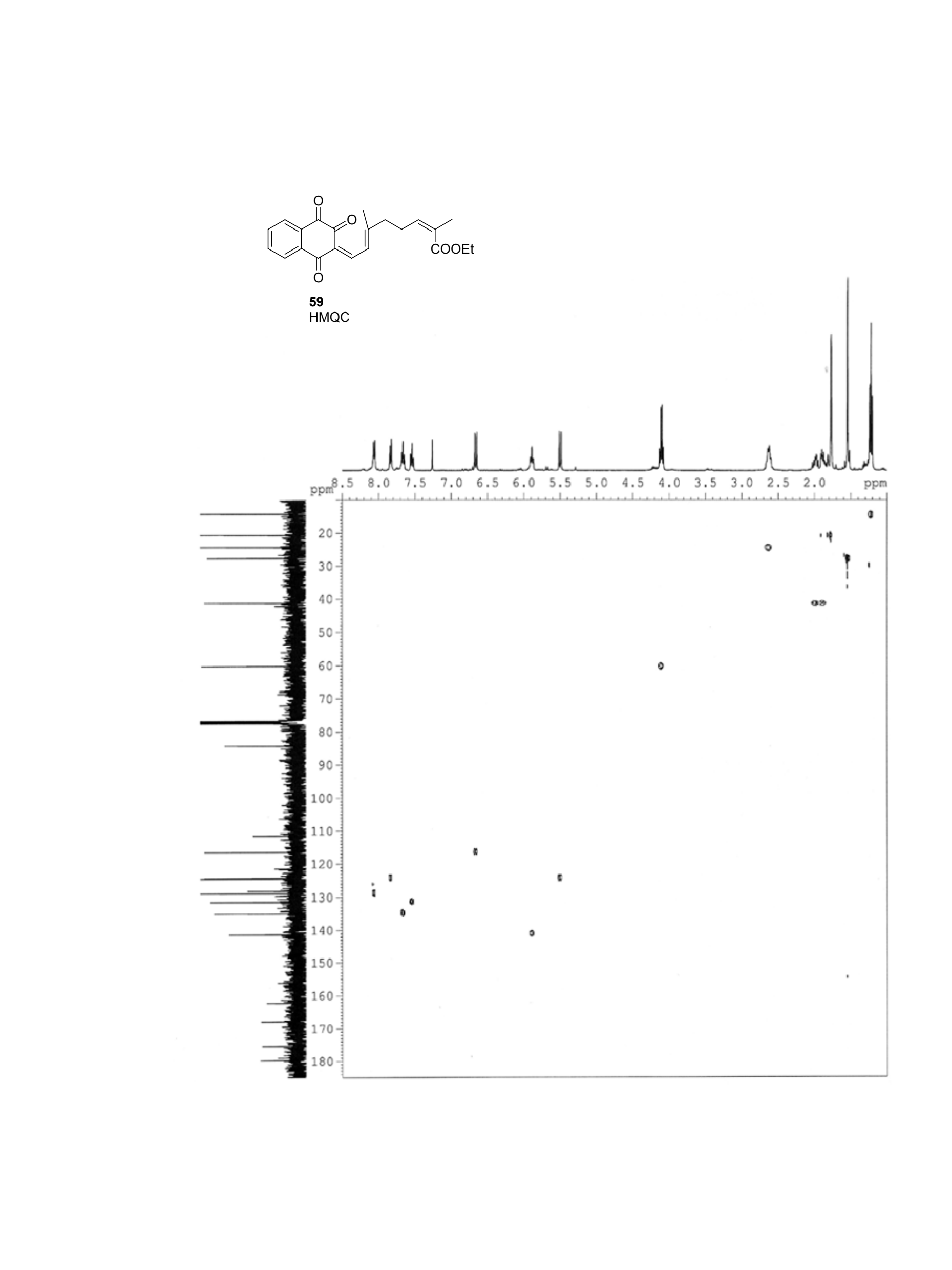

